\title{
THE VALUE OF ASSESSING UNCERTAINTY
}

\author{
A Dissertation \\ by \\ MUBARAK NASSER M ALDOSSARY \\ Submitted to the Office of Graduate and Professional Studies of \\ Texas A\&M University \\ in partial fulfillment of the requirements for the degree of \\ DOCTOR OF PHILOSOPHY
}

\begin{tabular}{|c|c|}
\hline \multirow{4}{*}{$\begin{array}{l}\text { Chair of Committee, } \\
\text { Committee Members, }\end{array}$} & Duane McVay \\
\hline & John Lee \\
\hline & Eduardo Gild \\
\hline & Bani Mallick \\
\hline & $\operatorname{Dan} \mathrm{H}$ \\
\hline
\end{tabular}

May 2016

Major Subject: Petroleum Engineering 


\begin{abstract}
Despite the perception of lucrative earnings in the oil industry, various authors have noted that industry performance is routinely below expectations. For example, the average reported return for the industry was around $7 \%$ in the $1990 \mathrm{~s}$, even though a typical project hurdle rate was at least $15 \%$. The underperformance is generally attributed to poor project evaluation and selection due to chronic bias. While a number of authors have investigated cognitive biases in oil and gas project evaluation, there have been few quantitative studies of the impact of biases on economic performance. Incomplete investigation and possible underestimation of the impact of biases in project evaluation and selection are at least partially responsible for persistence of these biases.

The objectives of this work were to determine quantitatively the value of assessing uncertainty or, alternatively, the cost of underestimating uncertainty. This work presents a new framework for assessing the monetary impact of overconfidence bias and directional bias (i.e., optimism or pessimism) on portfolio performance. For moderate amounts of overconfidence and optimism, expected disappointment (having realized NPV less than estimated NPV) was 30-35\% of estimated NPV for typical industry portfolios and optimization cases. Greater degrees of overconfidence and optimism resulted in expected disappointments approaching $100 \%$ of estimated NPV. Comparison of simulation results with expected industry performance in the 1990s, indicates that these greater degrees of overconfidence and optimism have been experienced in the industry.
\end{abstract}


The value of reliably quantifying uncertainty is in reducing or eliminating expected disappointment and expected decision error (selecting the wrong projects), which is achieved by focusing primarily on elimination of overconfidence; other biases are taken care of in the process. Elimination of expected disappointment will improve industry performance overall to the extent that superior projects are available and better quantification of uncertainty allows identification of these superior projects. 


\section{ACKNOWLEDGEMENTS}

I would like to thank my committee chair, Dr. McVay, for the countless number of hours and for the inspiration, and also my committee members, Dr. Lee, Dr. Gildin, and Dr. Mallick, for their guidance and support throughout the course of this research.

Thanks also to my mother and father for their encouragement, to my wife for her patience and love, and to my kids for bringing happiness to my face when I least expect it.

Finally, thanks to the Society of Petroleum Engineers for the copyright permission to reuse SPE-160189 “The Value of Assessing Uncertainty," doi:10.2118/160189-PA in this dissertation. Copyright 2014, Society of Petroleum Engineers Inc. Reproduced with permission of SPE. Further reproduction prohibited without permission. 


\section{TABLE OF CONTENTS}

Page

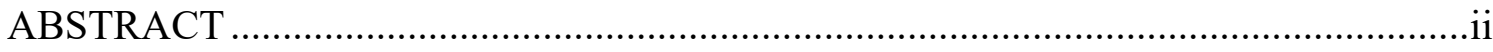

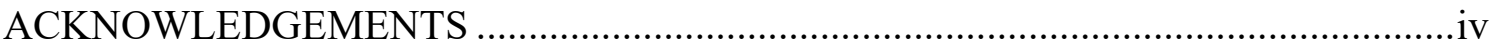

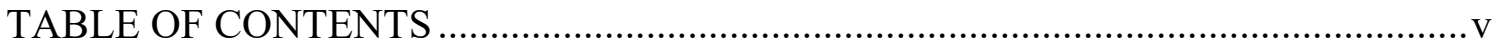

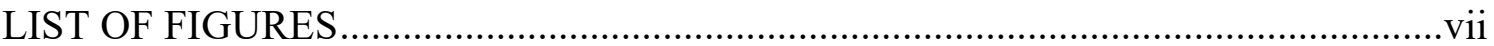

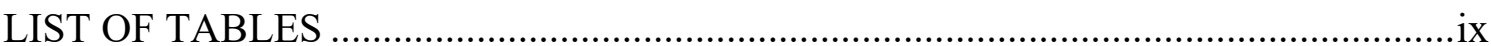

INTRODUCTION

PREVIOUS MODELING OF IMPACT OF BIASES ………………….....................

NEW FRAMEWORK FOR MODELING IMPACT OF BIASES ...................................12

Chronic Overconfidence and Optimism.................................................................. 12

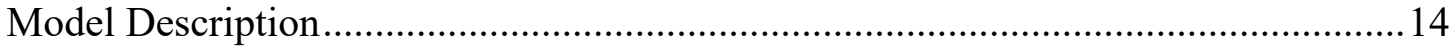

True project value distribution .........................................................................

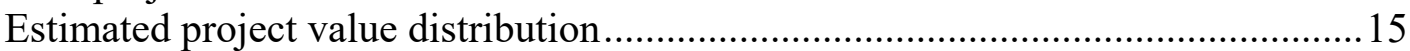

Intuitive description........................................................................................ 17

Quantitative Assessment of Impact of Biases..........................................................21

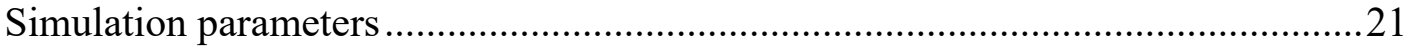

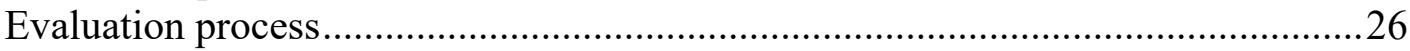

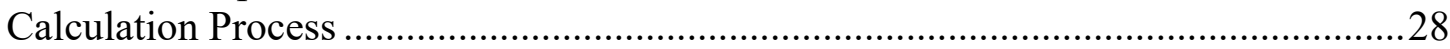

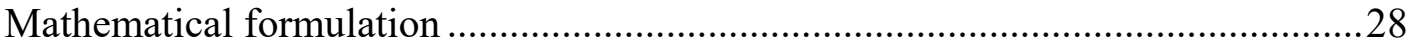

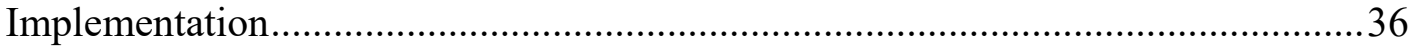

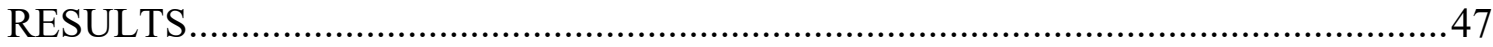

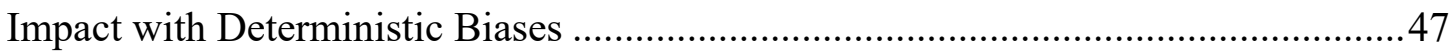

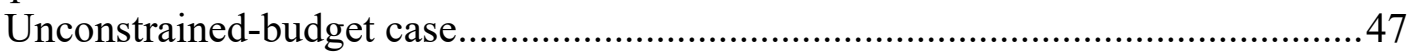

Constrained-budget case ............................................................................5

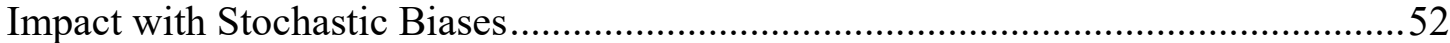

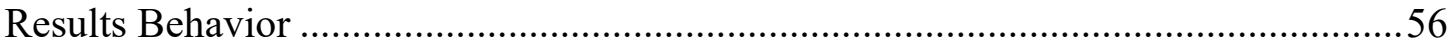

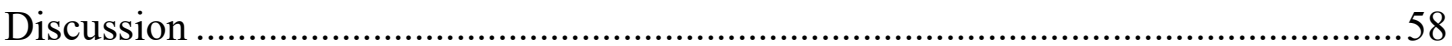

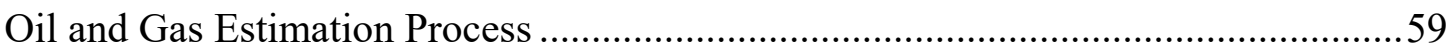

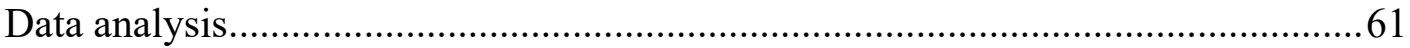




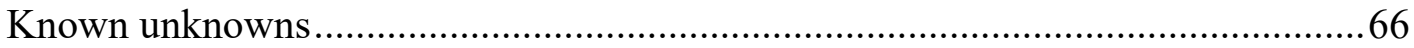

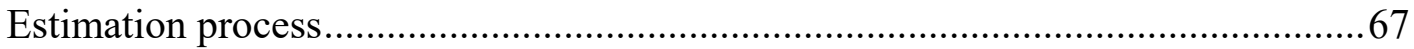

Sources of overconfidence and directional bias .............................................. 70

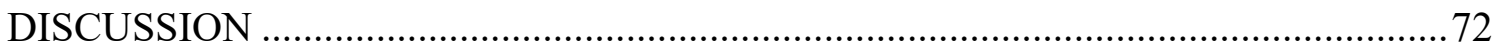

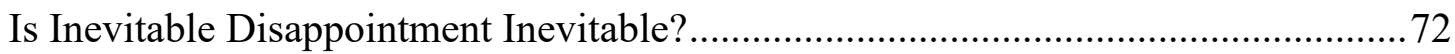

Relationship of Biases Impact to Industry Performance ........................................ 74

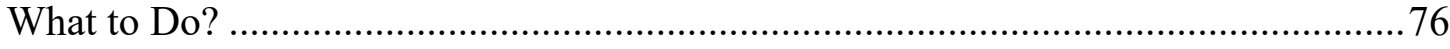

Measuring Confidence and Directional Bias ....................................................... 78

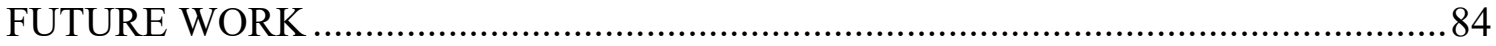

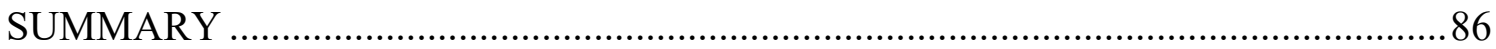

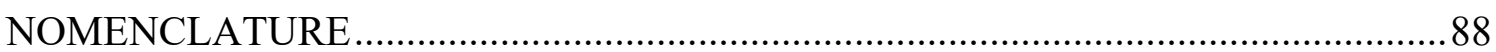

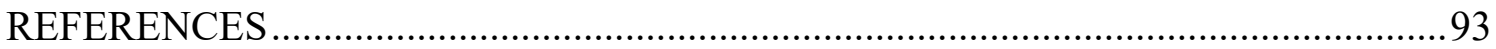




\section{LIST OF FIGURES}

Page

Fig. 1-Relationship between the estimated distribution (shaded) and the true distribution (unshaded) as a function of overconfidence and directional bias parameters for a value-based parameter such as NPV. The overconfidence parameter determines the fraction of explored probability while the bias parameter determines the direction.

Fig. 2-Professional responded with 32\% when asked for $90 \%$ ranges, which yields a confidence parameter value of 0.644 . Figure exaggerated for illustration (not drawn to scale).

Fig. 3-Lognormal distribution approximation to PVOCF (X-axis is in \$)

Fig. 4-Comparison between $\mathrm{P}(\mathrm{NPV}<0)$ for the three global portfolios. NPV in blue, CapEx*IE in green and PVOCF-CapEx in red. (X-axis in MM\$).

Fig. 5-Illustration for mathematical calculation for directional bias value of 0.5 and overconfidence value of 0.5 . (X-axis in $\mathrm{MM} \$$ )

Fig. 6-CDF of PVOCF for overconfidence and directional bias values of 0.5. $(\mathrm{X}$-axis in MM\$)

Fig. 7-Expected disappointment, unconstrained budget with deterministic bias. 48

Fig. 8-Expected decision error, unconstrained budget with deterministic bias. 50

Fig. 9-Expected disappointment, constrained budget with deterministic bias.

Fig. 10 - Expected decision error, constrained budget with deterministic bias.

Fig. 11-Expected disappointment, unconstrained budget with stochastic bias.

Fig. 12- Expected decision error, unconstrained budget with stochastic bias .55

Fig. 13- Expected disappointment, constrained budget with stochastic bias. 55

Fig. 14-Expected decision error, constrained budget with stochastic bias. 56

Fig. 15-Estimated NPV for high OC and DB. 57

Fig. 16-Realized NPV for high OC and DB 58 
Fig. 17-Production profile of six pilot wells in Coal Bed Methane prospect ...............60

Fig. 18-Field recovery factor per well decreases with smaller well-spacing, i.e. increased number of wells........................................................................62

Fig. 19- Gas content versus depth from wells in the same field..............................63

Fig. $21-$ Net pay versus depth for the five layers in the reservoir ............................... 64

Fig. 22-Cost of pipeline tie-in from surveyed wells in the same area .......................65

Fig. 23-Probability Density function result of four typical estimation processes based on well spacing (acres/well) ......................................................... 70 


\section{LIST OF TABLES}

Page

Table 1 - Summary of comparison to previous work 10

Table 2-Monte-Carlo simulation results for the percentage of true NPV less than zero for the approximated lognormal distribution of PVOCF. Highlighted row represents the selected case.....................................................................24

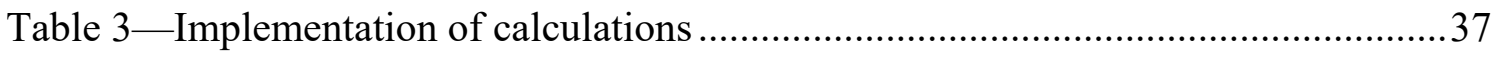

Table 4-Analysis of unconstrained budget estimation results .................................42

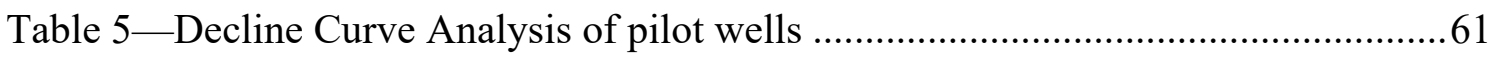

Table 6 - List of sources of uncertainty in example estimation process.......................66

Table 7-Capital expenditures (samples from one Monte Carlo iteration) ...................68

Table 8-Field production calculations (samples from one Monte Carlo iteration) .......68

Table 9-Operating cash flow calculations (sample from one Monte Carlo iteration) ...69 


\section{INTRODUCTION*}

A number of authors have documented the chronic underperformance of the oil and gas industry relative to expectations. Brashear et al. (2001) reported that the return on net assets by the largest U.S.-based E\&P companies was only 7\%, after selecting projects with hurdle rates of generally $15 \%$ or more, which were all financed with cost of capital generally in the range of 9-12\%. According to Rose (2004), in the last 20 years of the $20^{\text {th }}$ century, E\&P companies delivered only about half of predicted reserves. From 1990 to 2000 , the S\&P 500 had an average annual return of $16 \%$, almost double E\&P companies at 9\%. The top 25 international E\&P companies from 1996-2002 averaged only $11 \%$ return on exploration investment.

While performance may have improved in the last decade, this may be due more to high oil prices during this period rather than systematic improvement in business processes. It is even possible that high oil prices have caused the industry to relax and have made decision-making worse. Merrow (2011) reported that since 2003, the rate of success for megaprojects ( $>\$ 1$ billion) remained constant at about $50 \%$ for nonpetroleum projects, while the success rate for petroleum megaprojects declined from $50 \%$ to $22 \%$.

\footnotetext{
* Reproduced with permission of Society of Petroleum Engineers (SPE) to reuse SPE-160189 "The Value of Assessing Uncertainty," doi:10.2118/160189-PA. Copyright 2014, Society of Petroleum Engineers Inc. Further reproduction prohibited without permission.
} 
Why does the industry underperform? Brashear et al. (2001) attributed it, at least in part, to use of evaluation methods that do not account for the "full" uncertainty. Rose (2004) indicated that "by far, the dominant cause of E\&P underperformance is an internal problem...: Chronic bias in estimating key geotechnical parameters that control our evaluation of E\&P projects.” According to Begg et al. (2003), "Uncertainty is the underlying cause of the failure of many decisions to deliver their promised technical and economic metrics." Is the problem the uncertainty itself? This cannot be the case because uncertainty will always be present. Indeed, Leach (2006) says that uncertainty is the ultimate source of value in business. If not uncertainty itself, is the problem that our processes for decision making under uncertainty are suboptimal, or is it that we do a poor job of quantifying the uncertainty?

Capen (1976) summarized well the difficulty of assessing uncertainty. He conducted several experiments with petroleum engineers that demonstrated chronic overconfidence, or underestimation of uncertainty. For example, he showed that when asked to produce $90 \%$ confidence intervals, engineers on average produced ranges that corresponded to only $32 \%$ confidence intervals. He discussed the value of training (calibration) and application of probabilistic methods to improve uncertainty estimation. A number of authors have identified specific biases that affect estimation and decision making (Tversky and Kahneman, 1974; Virine, 2008; Welsh et al., 2007; Welsh et al., 2005). The authors claim that awareness of these biases and training can mitigate their effects and improve estimation. 
Many authors have written on the benefits of uncertainty quantification and probabilistic decision analysis processes. For example, Capen (1976) demonstrated that efforts to better define the range, or distribution, can result in a more accurate "best estimate" used in decision making. Bratvold and Begg (2010, pp. 86-88) show that quantifying variability (uncertainty) in potential outcomes is important because it can help you determine if the downside is so large that it could ruin you. Due to these and other papers and technology transfer efforts, Bickel and Bratvold (2007) show that there has been increased interest and use of probabilistic decision making processes in the oil and gas industry. However, a significant fraction of companies still use predominantly deterministic methods.

Despite the many papers and increased awareness and use of probabilistic methods, why is there not greater widespread adoption? Why does the industry apparently still underestimate uncertainty, routinely and significantly? Why is there little apparent application of look-backs and calibration of probabilistic forecasts to improve uncertainty quantification? Part of the answer to these questions is that some companies that have tried uncertainty quantification and decision analysis have experienced "uncertainty-induced confusion" due to incorrect application (Bickel and Bratvold, 2007). A greater contributor is lack of clear evidence that increased use of uncertainty quantification and decision analysis has led to significantly better decision making and overall industry performance. Most of the cited benefits of uncertainty quantification and probabilistic decision analysis processes have been qualitative in nature, as the couple mentioned above. There have been few quantitative studies. In a study outside of 
petroleum E\&P, Clemen and Kwit (2001) estimated that Eastman Kodak Company added value of $\$ 250$ million to $\$ 1$ billion from 178 decision-analysis projects performed for the company from 1990-1999. For the E\&P industry, Welsh et al. (2007) performed a quantitative analysis of the impact of three biases common to project evaluationoverconfidence, trust, and availability. They showed that the presence of these biases could result in true NPVs substantially less than estimated NPVs. However, the analysis was performed on a single-project basis and the overall impact on a portfolio was not assessed. Begg and Bratvold (2008) performed a quantitative analysis of the impact of prediction error on an expected basis at the portfolio level. However, they examined only the impact of the Optimizer's Curse systematic bias, which they found to have less impact than previously thought. With the dearth of quantitative studies, there is little evidence demonstrating that uncertainty quantification and probabilistic processes actually add value to the bottom line. This lack of evidence is one of the primary reasons many companies are reluctant to adopt these processes.

The objectives of this work were to determine quantitatively the value of assessing uncertainty or, alternatively, the cost of underestimating uncertainty. Can it really make a difference in the bottom line? Can it have a significant impact on company and industry financial performance? In this dissertation, the focus will not be on the different types and degrees of decision analysis processes available. Instead, the focus will primarily be on quantification of uncertainty, more specifically, reliable quantification of uncertainty. Many believe that simply conducting a probabilistic analysis is enough, and they do not understand nor appreciate the value of quantifying 
the true uncertainty. In the remainder of this work, some previous approaches will be reviewed, a new framework for modeling the impact of quantifying uncertainty will be presented, the results of numerical experiments will be presented and analyzed, and then the implications and application of the findings will be discussed. 


\section{PREVIOUS MODELING OF IMPACT OF BIASES*}

This section will present a brief summary of the Begg and Bratvold (2008) study of the Optimizer's Curse, or Inevitable Disappointment, because this work will build upon their modeling part. Begg and Bratvold conducted unconstrained-budget and constrainedbudget selections from typical O\&G Global Portfolios. The portfolios each consisted of 100 projects and were sampled from distributions designed to model the project alternatives available to a typical O\&G company. For each project in the unconstrainedbudget selection, they first sampled from a global distribution to determine the "true" expected NPV, or EV, that would be obtained if the company had unlimited time, money, and computational ability (unlimited-resources assessment). The authors did not define the "true" uncertainty (e.g., standard deviation or complete distribution) of the project, only the true EV. The authors then sampled from a second global distribution to get the standard deviation that is used to construct a normal distribution (with mean equal to the true $\mathrm{EV}$ ) that represents the estimated $\mathrm{EV}$ distribution that would result from typical industry limited-resources probabilistic assessments. They then sampled from this project normal distribution to obtain the estimated EV for the particular project assessment. Again, because it was not needed in their modeling, the authors did not quantify the estimated project uncertainty (e.g., standard deviation or complete

\footnotetext{
* Reproduced with permission of Society of Petroleum Engineers (SPE) to reuse SPE-160189 "The Value of Assessing Uncertainty," doi:10.2118/160189-PA. Copyright 2014, Society of Petroleum Engineers Inc. Further reproduction prohibited without permission.
} 
distribution) in their limited-resources assessments; they quantified only the estimated EV.

This process was repeated to determine true and estimated EVs for each of the 100 project alternatives in the portfolio. In the unconstrained-budget selection, projects were accepted if the EV $>0$, and rejected otherwise. The selection was done twice, first based on true EVs and second based on estimated EVs. From this information, the authors then determined the disappointment and decision error for the portfolio. The disappointment is determined from the portfolio selected using the estimated EVs, and is defined as the estimated expected value of this portfolio (estimated portfolio value) minus the true expected value of this same portfolio (realized portfolio value). Disappointment can be either positive or negative. The decision error is defined as the true expected value of the portfolio selected using the true EVs (best possible portfolio value) minus the true expected value of the portfolio selected using the estimated EVs (realized portfolio value). Thus, the decision error is always positive and it is defined as that portion of disappointment that results from selecting the wrong projects. The remaining portion of disappointment, estimated portfolio value minus the best possible portfolio value, is the estimation error. This selection process was repeated for many 100-project portfolios in a Monte Carlo simulation to determine the expected disappointment, ED, and the expected decision error, EDE.

The process was similar for the authors' constrained-budget selection. However, instead of modeling a single random variable, NPV, the authors modeled two random variables, capital expenditure (CapEx) and investment efficiency (IE = NPV/CapEx). 
The projects were ranked by decreasing IE and then successively selected until the CapEx budget was reached (taking an appropriate percentage of the last project selected to fill the budget). NPV was backed out from IE and CapEx for purposes of determining expected disappointment and expected decision error.

Parameters specified by Begg and Bratvold (2008) were as follows:

1) Unconstrained-budget selection used only NPV of projects:

a) The true expected NPV (EV) is modeled as a shifted log-normal distribution (mean 600MM, SD 300MM, truncated from the right at 1,800MM, and then shifted $-300 \mathrm{MM})$. The maximum NPV is $1,500 \mathrm{MM}$ based on the order of the truncation and shift.

b) Variability (standard deviation) of the estimated EV about the true EV is a Pert distribution (min 50MM, most likely 100MM, max 200MM).

2) Constrained-budget selection used CapEx and IE parameters, from which NPV was back-calculated.

a) True expected IE is a shifted log-normal distribution (mean 0.5, SD 0.3, truncated from the right at 2.3, and then shifted -0.3).

b) Variability of estimated IE is a Pert distribution ( $\min 0.05$, most likely $0.1, \max$ $0.2)$.

c) True expected CapEx is a shifted log-normal distribution (mean 600MM, SD 600MM, and shifted +100MM).

d) Distributions of estimated EV about the true EV for CapEx and IE are normal distributions. 
e) IE and CapEx are independent (uncorrelated).

f) Maximum CapEx budget is $\$ 5$ billion.

In the process of reproducing the experiments of Begg and Bratvold, it was noticed that the variability of CapEx was not specified in their paper, and therefore a Pert distribution (min 50MM, most likely 100MM, max 200MM) was used based upon personal communication with the authors. One million iterations in Monte Carlo simulations were used, whereas the authors did not specify the number of Monte Carlo iterations they used. The expected disappointment was calculated as a percentage of true NPV (ED\%T) in this work by taking the expectation of the percent disappointments from each of the Monte-Carlo iterations, $E D \% T=E\left(\frac{E s t N P V-T N P V}{T N P V}\right)$, as opposed to calculating it from the expected total NPVs, $E D \% T=\frac{E(E s t N P V)-E(T N P V)}{E(T N P V)}$, as the authors apparently did in the sample calculation outlined in their paper. Similar calculations were made for expected decision error as percent of true NPV (EDE\%T). Despite these differences, their expected disappointment and expected decision error values were matched to within $0.1-0.2 \%$ for both the unconstrained-budget and constrained-budget cases (Table 1). 
Table 1-Summary of comparison to previous work

\begin{tabular}{|l|l|l|l|l|}
\hline Case type & $\underline{\text { Parameter }}$ & $\underline{\underline{T h i s}}$ & $\underline{\text { SPE }}$ & $\underline{\text { Using second global }}$ \\
\hline \multirow{2}{*}{$\begin{array}{l}\text { Unconstrained } \\
\text { budget }\end{array}$} & Expected Disappointment & $4.9 \%$ & $\underline{116525}$ & portfolio distribution \\
\cline { 2 - 5 } & Expected Decision Error & $2.2 \%$ & $2.1 \%$ & $4.07 \%$ \\
\hline $\begin{array}{l}\text { Constrained } \\
\text { budget }\end{array}$ & Expected Disappointment & $5.9 \%$ & $6.1 \%$ & \\
\cline { 2 - 5 } & Expected Decision Error & $2.8 \%$ & $2.9 \%$ & \\
\hline
\end{tabular}

Begg and Bratvold (2008) described the global portfolios differently for the two cases they ran. They described the first global portfolio in terms of NPV for the unconstrained-budget case and the second global portfolio in terms of CapEx and IE for the constrained-budget case. These descriptions do not result in identical properties for the two global portfolios. To test the sensitivity of expected disappointment and expected decision error to properties of the global portfolio, an unconstrained-budget selection was carried out using the second portfolio description, i.e., the one in terms of CapEx and IE used in the second case. Both ED\%T and EDE\%T for the unconstrainedbudget case using the second portfolio description were approximately double the $\mathrm{ED} \% \mathrm{~T}$ and EDE\%T obtained using the first portfolio description (Table 1), with ED\%T approaching 10\% and EDE\% $\%$ over 4\%. This difference indicates that ED\%T and $\mathrm{EDE} \% \mathrm{~T}$ are sensitive to properties of the global portfolio, so a way to characterize the global portfolio was needed. While not completely descriptive, a relatively simple measure is the percentage of projects in the global portfolio that have true expected 
NPV, or EV, less than zero. Analysis showed that the first portfolio, described in terms of NPV, has approximately $11 \%$ of the projects with true EV less than zero, while the second portfolio, described in terms of CapEx and IE, has $26 \%$ of the projects with true expected NPV less than zero (Fig. 4). Thus, the second portfolio is more negative, which helps explain why it experiences greater $\mathrm{ED} \% \mathrm{~T}$ and $\mathrm{EDE} \% \mathrm{~T}$. These results indicate that for global portfolios that are even more negative than these, $\mathrm{ED} \% \mathrm{~T}$ and $\mathrm{EDE} \% \mathrm{~T}$ could potentially be more significant than that reported by Begg and Bratvold. 


\section{NEW FRAMEWORK FOR MODELING IMPACT OF BIASES*}

\section{Chronic Overconfidence and Optimism}

Much has been written about the biases that affect judgment and estimation, and authors have identified many different biases. Reviewing these biases reveals that they tend to affect:

1) the uncertainty, or variability, of the estimate (usually in the direction of overconfidence, or underestimation of uncertainty),

2) the central tendency of the estimate (usually in the direction of optimism), or

3) both the uncertainty and central tendency of the estimate.

It is well established that chronic overconfidence is a problem of nearly everyone, including oil and gas industry professionals. This was reported by Tversky and Kahneman (1974), Capen (1976), and other authors. Little evidence shows there has been significant progress in reducing the overconfidence problem since these papers were written in the 1970s. For example, Ben-David et al. (2013) surveyed top U.S. financial executives over a 10-year period, yielding 12,500 probability distributions of future stock market returns. They found the CFO's were severely overconfident; realized market returns were within predicted $80 \%$ confidence intervals only $33 \%$ of the time.

This overconfidence was strongly correlated to overconfidence in their own-firm returns.

\footnotetext{
* Reproduced with permission of Society of Petroleum Engineers (SPE) to reuse SPE-160189 "The Value of Assessing Uncertainty," doi:10.2118/160189-PA. Copyright 2014, Society of Petroleum Engineers Inc. Further reproduction prohibited without permission.
} 
Another serious problem in oil and gas project evaluation is chronic optimism. Optimism is the tendency to ignore or not consider possible negative outcomes, or the tendency to give greater weight to possible positive outcomes than possible negative outcomes. Psychologists have demonstrated a general tendency for unrealistic optimism; people underestimate the likelihood that negative events will happen to them and overestimate the likelihood that positive events will happen to them (Weinstein, 1980). Some studies suggest that people are more optimistically biased under conditions of greater uncertainty and when the outcomes of their predictions will be revealed further in the future (Armor and Taylor, 2002). Many authors allude to or provide evidence of optimism bias in the oil and gas industry (Begg and Bratvold, 2008; Campbell et al., 2001; Merrow, 2011; Smith and Winkler, 2006).

Overconfidence and optimism work together. We are overconfident because we are optimistic and fail to see all the downsides. We are optimistic because we are overconfident and fail to see all the downsides. It may be possible to be overconfident without being optimistic if one neglects upsides and downsides equally, although the evidence suggests that this is not common. However, it is unlikely that we can be optimistic without being overconfident; if we are considering all possible outcomes and are thus not overconfident, then we are considering both the upsides and the downsides and are thus not optimistic. Thus, it appears that overconfidence is the first cause and the more significant of the two biases. Review of the oil and gas literature does not show any systematic quantitative studies of overconfidence and optimism in combination. 


\section{Model Description}

In this section the mathematical model used to assess the value of quantifying uncertainty will be described. The model is relatively simple and is based on the premise that all the biases that affect oil and gas project evaluation can be boiled down to two primary biases — overconfidence and optimism (or more generally, directional bias). The model describes the relationship between the true project value distribution(s) (e.g., NPV) and the estimated project value distribution(s) in terms of an overconfidence parameter and a directional bias parameter.

\section{True project value distribution}

As with Begg and Bratvold (2008) and others, the "true" project value distribution is assumed to be the distribution that would result from an unlimited-resources assessment, i.e., that would be obtained if the company had unlimited time, money, and computational ability. Furthermore, it is clarified that the unlimited-resources assessment is based upon existing data; i.e., the unlimited time and money cannot be used to run additional tests, drill more wells, or acquire additional data for the project. Questions such as whether a "true" project distribution exists, if it is unique, and what it means are further discussed by Smith and Winkler (2006). It is assumed here that a "true" project distribution exists, but that it is not necessarily the same for every individual or company. Since uncertainty is personal (different individuals and companies have different knowledge and assessment processes, and thus can have different uncertainty for a particular project), different individuals and companies can have different but reliable "true" unlimited-resources project value distributions. Reliable means that the 
true distributions are perfectly calibrated. That is, if many similar evaluations were to be conducted, the frequencies of realized outcomes would correspond perfectly with the probabilities of those outcomes, for all outcomes over the entire range of probability from 0 to 1 .

\section{Estimated project value distribution}

This is the distribution that would result from a typical industry limited-resources probabilistic assessment, and would include the biases present in typical oil and gas project evaluations.

\section{Overconfidence}

First, the impact of overconfidence on the estimated project value distribution will be considered in the model. Overconfidence is defined as a parameter that ranges from 0 to

1. This value specifies the fraction of the true distribution (or total probability) that is not sampled in the limited-resources assessment. Thus, an overconfidence value of 0 means the entire true distribution is sampled in the limited-resources assessment. In such a case, all possible outcomes are considered, no biases are present, and the limited-resources project value distribution is exactly the same as the true distribution. Overconfidence values greater than 0 indicate that only a subset of the true distribution is sampled, or quantified. This means that some possible outcomes are not considered in the limitedresources assessment. This results in a narrowing of the distribution, which is overconfidence. The assumption here is that the extreme, least-likely possibilities, or "black swans" (Taleb, 2007), are not considered, and we thus simply truncate the distributions at the tails (Fig. 1). While this is done for simplicity, it is believed to be not 
far from reality. Assuming for the moment no optimism or pessimism (directional bias), the distribution will be truncated by the same amount of probability (or area under the curve) from each end. The more overconfident the evaluator is, the larger the value of the overconfidence parameter, and the narrower the truncated distribution is (Fig. 1, diagrams with directional bias parameter equal to zero). A value of 0.5 means the evaluator will consider only the outcomes representing the most-likely $50 \%$ of the total probability (area under the curve), truncating $25 \%$ of the total probability from the low end and $25 \%$ of the total probability from the high end. A confidence parameter value of one indicates no range at all, or a deterministic single estimate at the median of the probability distribution.

\section{Directional Bias}

Next, the impact of optimism or pessimism (directional bias) on the estimation will be considered. Directional bias is defined as a parameter that specifies the location of the estimated distribution within the true distribution. The directional bias parameter value ranges from -1 to 1 , with -1 denoting complete pessimism and 1 specifying complete optimism. Fig. 1 shows, for normal distributions for a value-based parameter such as NPV, the portion of the true distribution that is sampled for various combinations of the confidence parameter and the directional bias parameter. A directional bias of 1 indicates complete optimism, which means that only the most optimistic outcomes are considered in the limited-resources assessment. In this case, there is no truncation of the distribution from the high end; all of the truncation is from the low end. A directional bias of -1 indicates complete pessimism, which means that only the most pessimistic outcomes are 
considered in the limited-resources assessment. In this case, there is no truncation of the distribution from the low end; all of the truncation is from the high end. In this model, there can be directional bias only if there is overconfidence, i.e., confidence parameter $>$ 0 . Directional bias has no impact for confidence parameter $=0$, since in this case the estimated distribution is the same as the true distribution.

\section{Intuitive description}

As mentioned above, a directional bias equal to zero indicates neither optimism nor pessimism, in which case truncation of the true distribution occurs equally from both ends. Linear interpolation is used to obtain the fractions of area truncated from each end for other values of directional bias. For example, for directional bias of $0.5,75 \%$ of the total probability, or area, that is truncated (which depends on the value of the confidence parameter) is truncated from the left end of the true distribution and $25 \%$ is truncated from the right end of the distribution. Thus, for a confidence parameter value of 0.5 , $37.5 \%$ of the probability (area) would be removed starting from the left and $12.5 \%$ of the probability would be removed starting from the right end of the distribution (Fig. 1). Truncation for a cost-based parameter such as CapEx would be in the opposite direction. For example, directional bias of 1 (optimism) would result in all the truncation being from the high end, shifting the estimated distribution to lower CapEx values. 


\begin{tabular}{|c|c|c|c|}
\hline & Confidence $=0.10$ & Confidence $=0.50$ & Confidence $=0.90$ \\
\hline \multicolumn{4}{|l|}{$\begin{array}{l}\text { Directional } \\
\text { Bias }=1.0\end{array}$} \\
\hline & Est $\mathrm{EV}=0.2$ & Est $\mathrm{EV}=0.8$ & Est $\mathrm{EV}=1.8$ \\
\hline \multicolumn{4}{|l|}{$\begin{array}{l}\text { Directional } \\
\text { Bias }=0.5\end{array}$} \\
\hline & Est $\mathrm{EV}=0.1$ & Est $\mathrm{EV}=0.3$ & Est $\mathrm{EV}=0.6$ \\
\hline \multicolumn{4}{|l|}{$\begin{array}{l}\text { Directional } \\
\text { Bias }=0.0\end{array}$} \\
\hline & Est $\mathrm{EV}=0.0$ & Est $\mathrm{EV}=0.0$ & Est $\mathrm{EV}=0.0$ \\
\hline \multicolumn{4}{|l|}{$\begin{array}{l}\text { Directional } \\
\text { Bias }=-0.5\end{array}$} \\
\hline & Est $E V=-0.1$ & Est $\mathrm{EV}=-0.3$ & Est $E V=-0.6$ \\
\hline \multicolumn{4}{|l|}{$\begin{array}{l}\text { Directional } \\
\text { Bias }=-1.0\end{array}$} \\
\hline & Est $E V=-0.2$ & Est $E V=-0.8$ & Est $\mathrm{EV}=-1.8$ \\
\hline
\end{tabular}

Fig. 1-Relationship between the estimated distribution (shaded) and the true distribution (unshaded) as a function of overconfidence and directional bias parameters for a value-based parameter such as NPV. The overconfidence parameter determines the fraction of explored probability while the bias parameter determines the direction.

These truncated distributions are the limited-resources, "estimated" project distributions. Fig. 1 also shows, for each case, the expected value of the estimated distribution that results from truncation of a true standard normal distribution with mean of zero and standard deviation of 1 . The expected values from these truncated 
distributions are used in Monte Carlo simulations to calculate expected disappointment and expected decision error.

It is of interest to compare values of this confidence parameter to other measures of overconfidence (or underestimation of uncertainty) presented in the literature. For example, Capen (1976) demonstrated that when many O\&G professionals were requested to provide $90 \%$ confidence intervals for a variety of general-knowledge estimations, the professionals gave ranges that encompassed the true value only $32 \%$ of the time. That is, the requested $90 \%$ ranges were in fact only $32 \%$ ranges. Translating those numbers into the new framework yields a confidence parameter of 0.644 , i.e. ( 1 35.6\%), as shown in Fig. 2. To explain the calculation, unshaded areas under the curve imply possibilities not considered due to overconfidence. A typical professional with such a confidence value (0.644) will only have a perceived possible range of $35.6 \%$, and the professionals gave $90 \%$ of that $35.6 \%$, which is $32 \%(0.9 \times 35.6 \%)$-what Capen measured. A general formula for converting similar quantities is: confidence value $=$ $1-\frac{\text { Range }_{\text {received }}}{\text { Range }_{\text {requested }}}$. 


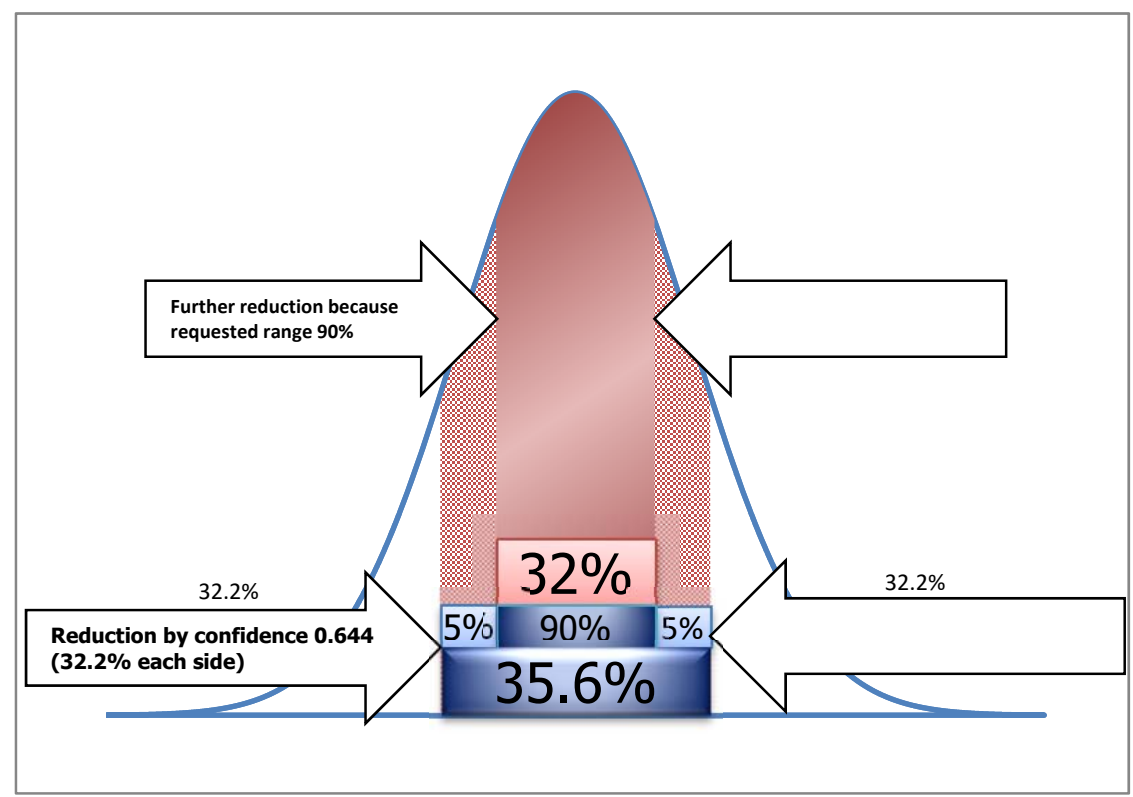

Fig. 2-Professional responded with $32 \%$ when asked for $90 \%$ ranges, which yields a confidence parameter value of $\mathbf{0 . 6 4 4}$. Figure exaggerated for illustration (not drawn to scale).

In Welsh et al. (2007), the authors requested $80 \%$ confidence ranges for O\&Grelated estimations, but instead, received on average only $50 \%$ ranges. This corresponds to a confidence parameter value of 0.375 . Smaller confidence value for familiar tasks is expected and reproduced by others (Block and Harper, 1991).

In this study, the investigated values of the confidence parameter will range from 0 to 1 , i.e., all possible values. The investigated values of directional bias will range from 1 (complete optimism) to -1 (complete pessimism). The negative directional bias (pessimism) will be included even though evidence suggests optimism is much more common than pessimism in oil and gas project evaluation. Initially, discrete values of confidence and directional bias parameters are used to determine their effects on 
expected disappointment and decision error. Later in this work, additional scenarios are investigated to simulate human bias behavior which might vary from one project to the next, and also from one portfolio estimator to the next. Normal distributions were used in Fig. 1 for illustration but the analysis will use lognormal distributions as will be explained in the parameters section.

\section{Quantitative Assessment of Impact of Biases}

It would be of interest to first reproduce Begg and Bratvold (2008) work on the Optimizer's Curse with the new framework that was just introduced. However, it is not possible to do this directly because the authors specified only the true EV and did not specify the true project uncertainty (e.g., standard deviation), while both the true EV and the true project uncertainty are required in this framework. In this quantitative analysis, the starting point for the portfolio description will be the one employed by Begg and Bratvold for their constrained-budget selection, described in terms of CapEx and IE. A model for the true project uncertainty is added and other modifications were made, which are described in the next section.

\section{Simulation parameters}

The most significant change made was in the two random variables used to describe project economic performance. Instead of using CapEx and IE, which are inversely correlated because CapEx is the denominator of IE, CapEx and the present value of the operating cash flow (PVOCF) are chosen to be used. PVOCF includes all cash inflows and outflows except for CapEx and is thus equal to NPV plus CapEx. CapEx and 
PVOCF are modeled as independent random variables. While there may be some correlation between these variables in actual projects, since PVOCF in general represents the revenue side of the project (dominated by production and prices) while CapEx in general represents the development cost side of the project, there will be much less correlation between CapEx and PVOCF as there will be between CapEx and IE. CapEx and PVOCF distributions for the pool of 100 projects to select from were determined by sampling means and standard deviations from global distributions.

\section{CapEx}

For mean true CapEx, the same global distribution as Begg and Bratvold will be sampled, a lognormal distribution with mean $=\$ 600 \mathrm{MM}, \mathrm{SD}=\$ 600 \mathrm{MM}$ and then shifted positively by $\$ 100 \mathrm{MM}$. The standard deviation of true CapEx will be defined relative to the true expected value. Thus, large projects will have large uncertainty, and small projects will have smaller uncertainty. To obtain each project's CapEx standard deviation, the true expected CapEx is multiplied by a fraction sampled from a Pert distribution with minimum 0.3 , mode 0.8 , and maximum 1.3 . With a mean and standard deviation determined from these global distributions, the true CapEx for each project is modeled with a lognormal distribution.

\section{PVOCF}

Since there was not a previous model of PVOCF to start with, it was back-calculated from the previous CapEx and IE model. Since NPV by definition is $I E \times \operatorname{CapEx}$, and PVOCF by definition is PVOCF $=N P V+$ CapEx, then PVOCF $=I E \times C a p E x+$ CapEx. The first term is quite simple because multiplication or division of lognormal 
distributions gives a simple lognormal distribution form: $\log -\mathcal{N}\left(\mu_{\text {CapEx }}, \sigma_{\text {CapEx }}^{2}\right) \times$ $\log -\mathcal{N}\left(\mu_{I E}, \sigma_{I E}^{2}\right)=\log -\mathcal{N}\left(\mu_{\text {CapEx }}+\mu_{I E}, \sigma_{\text {CapEx }}^{2}+\sigma_{I E}^{2}\right)$. However, the addition and subtraction of two lognormal distributions does not have a closed form, and therefore must be approximated numerically. Using 10 million Monte-Carlo iterations, PVOCF was estimated by a lognormal distribution with mean $=\$ 746 \mathrm{MM}, \mathrm{SD}=\$ 731 \mathrm{MM}$ and then shifted positively by $\$ 87 \mathrm{MM}$. The fit between this approximated lognormal distribution and the randomly sampled numbers is shown in Fig. 3, where the red curve is the fitted lognormal distribution and the black rectangles are a histogram of the output from the Monte-Carlo simulation.

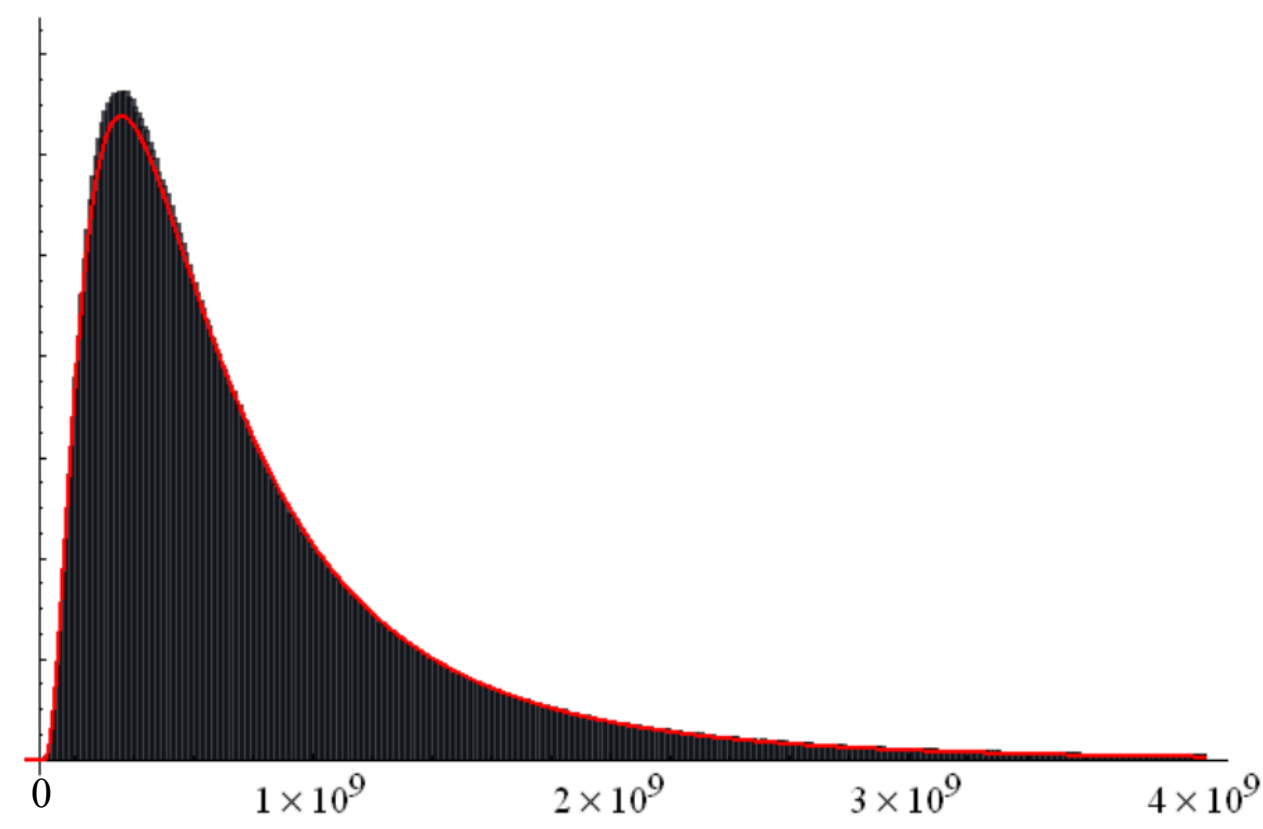

Fig. 3-Lognormal distribution approximation to PVOCF (X-axis is in \$) 
Then, the positive shift was adjusted until the global portfolio approximately matched the properties of the global portfolio from the previous CapEx and IE model, ending up with a global portfolio having $28 \%$ of the projects with true expected NPV less than zero. Keeping in mind that Begg and Bratvold's model had 26\% of projects with true expected NPV less than zero, the results of the Monte-Carlo simulation are presented in Table 2 with highlighted row for the selected case.

Table 2-Monte-Carlo simulation results for the percentage of true NPV less than zero for the approximated lognormal distribution of PVOCF. Highlighted row represents the selected case.

\begin{tabular}{|c|c|c|c|c|c|c|}
\hline \multicolumn{2}{|c|}{ Mean (\$MM) } & \multicolumn{2}{|c|}{ SD(\$MM) } & \multicolumn{2}{|c|}{ Shift(\$MM) } & \multirow{2}{*}{$\begin{array}{r}\text { TNPV }<0 \\
43 \%\end{array}$} \\
\hline$\$$ & 750.00 & $\$$ & 750.00 & $\$$ & 100.00 & \\
\hline$\$$ & 750.00 & $\$$ & 750.00 & $\$$ & 200.00 & $35 \%$ \\
\hline$\$$ & 750.00 & $\$$ & 750.00 & $\$$ & 300.00 & $28 \%$ \\
\hline$\$$ & 750.00 & $\$$ & 750.00 & $\$$ & 400.00 & $23 \%$ \\
\hline$\$$ & 750.00 & $\$$ & 750.00 & $\$$ & 500.00 & $18 \%$ \\
\hline$\$$ & 750.00 & $\$$ & 750.00 & $\$$ & 600.00 & $15 \%$ \\
\hline
\end{tabular}

The global distribution from which the mean true PVOCF is sampled will be simplified to a lognormal distribution with mean $=\$ 750 \mathrm{MM}, \mathrm{SD}=\$ 750 \mathrm{MM}$ and then shifted positively by $\$ 300 \mathrm{MM}$, while the global distribution from which the relative standard deviation of true PVOCF is sampled will be similar to the one used for relative standard deviation of true CapEx, which is a Pert distribution with minimum 0.3, mode 0.8 , and maximum 1.3. Again, the random value sampled from this distribution will be multiplied by the true value of PVOCF to get the standard deviation of PVOCF in each 
project. With a mean and standard deviation determined from these global distributions, the true PVOCF for each project is modeled with a lognormal distribution.

Fig. 4 shows a comparison between the three discussed global portfolio distributions. The first one, NPV in blue, was used in the unconstrained budget evaluation in Begg and Bratvold (2008) and had an average of $11 \%$ on projects with $\mathrm{NPV}<0$. The second one, IE * CapEx in green, was used in the Begg and Bratvold (2008) constrained budget estimation and had an average of $26 \%$ of projects NPV $<0$. The third one, PVOCF-CapEx was just proposed and was selected to have an average of $28 \%$ of projects with $\mathrm{NPV}<0$.

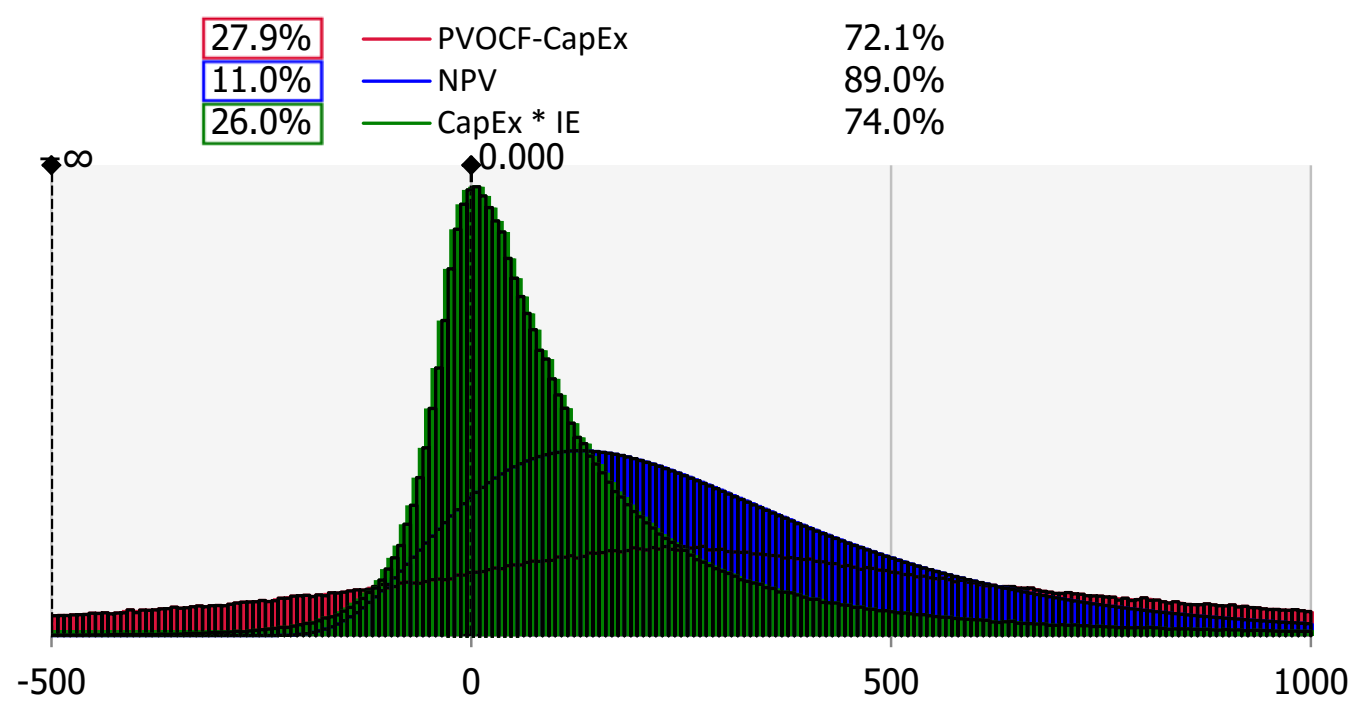

Fig. 4-Comparison between $\mathrm{P}(\mathrm{NPV}<0)$ for the three global portfolios. NPV in blue, CapEx*IE in green and PVOCF-CapEx in red. (X-axis in MM\$) 


\section{Evaluation process}

Confidence and directional bias parameters will be applied to the true distributions for CapEx and PVOCF to obtain estimated distributions for CapEx and PVOCF. Although CapEx and PVOCF could have been chosen to bias differently, in this modeling the same amount of bias was applied to both CapEx and PVOCF. For example, in cases with overconfidence of 0.5 , the overconfidence value of 0.5 will be applied to both models of CapEx and PVOCF. Directional bias is also applied equally, although estimated CapEx and PVOCF shift in opposite directions for the same value of directional bias. For example, in cases with optimism (directional bias $>0$ ), the estimated distribution for PVOCF is shifted in the positive direction while the estimated distribution for CapEx is shifted in the negative direction.

The framework for modeling biases was illustrated earlier using normal distributions (Fig. 1). Applying overconfidence with no directional bias preserves the mean, median and mode for normal distributions. However, lognormal distributions are used for true CapEx and true PVOCF for each potential project. Since truncating lognormal distributions cannot preserve both the mean and the median, this leads to the question of what overconfidence with no directional bias means for lognormal distributions. The mean could have been preserved, in which case the estimated EV would be the same as the true EV for overconfidence with no directional bias. However, it is more appropriate and realistic to truncate the same amount of probability, area under the curve, from each end of the distribution, which preserves the median of the lognormal distribution but results in a lower mean, or expected value. Since the 
estimated expected value is used in project selection, this means that being overconfident but neither optimistic nor pessimistic can result in non-ideal project selection and, thus, disappointment. It is difficult to predict exactly what the impact will be, given the interplay between CapEx and PVOCF, but this result could have important implications in project selection under uncertainty.

Finally, in the remainder of this work, ED and EDE are calculated as a percentage of the estimated portfolio value $(\mathrm{ED} \% \mathrm{E}$ and $\mathrm{EDE} \% \mathrm{E})$ rather than the realized portfolio value as was done earlier. It is more useful to define percent disappointment and decision error in terms of a quantity that is known ahead of time (estimated portfolio value) rather than a quantity that is unknown for some time in practice (realized portfolio value). In addition, for situations with large disappointment (which will arise later in this work), $\mathrm{ED}$ and $\mathrm{EDE}$ as a percentage of realized portfolio value (ED\%T and $\mathrm{EDE} \% \mathrm{~T})$ go to infinity as realized portfolio value goes to zero (realistic), while ED and EDE as a percentage of estimated portfolio value $(\mathrm{ED} \% \mathrm{E}$ and $\mathrm{EDE} \% \mathrm{E})$ go to infinity as estimated portfolio value goes to zero (less interest in this case, because such portfolios will less likely be selected).

The complete parameter set for both unconstrained-budget and constrainedbudget portfolio selection simulations is:

1) True portfolio description uses CapEx and PVOCF parameters, from which NPV and IE are back-calculated.

2) True expected CapEx is sampled from a shifted log-normal distribution (avg 600MM, SD 600MM, and then shifted 100MM). 
3) Standard deviation of true CapEx is relative to expected true CapEx, with fraction sampled from a Pert distribution (min 0.3, mode 0.8, max 1.3).

4) True expected PVOCF is sampled from a shifted log-normal distribution (avg 750MM, SD 750MM, and then shifted 300MM).

5) Standard deviation of true PVOCF is relative to expected true PVOCF, with fraction sampled from a Pert distribution (min 0.3, mode 0.8, $\max 1.3)$.

6) Individual-project true CapEx and PVOCF are lognormal distributions.

7) CapEx and PVOCF are sampled independently (uncorrelated).

8) Maximum CapEx budget is $\$ 5$ billion.

\section{Calculation Process}

\section{Mathematical formulation}

This section will outline the steps required to formulate the truncated distribution that models overconfidence and directional bias of an estimator. The equations will be accompanied by an example that represents the biased estimation of the most likely PVOCF with 0.5 overconfidence and 0.5 directional bias. The first part of modeling a specific directional bias parameter and a confidence parameter is to consider the area under the curve that will be explored by the estimator. Let us call the bounds of this investigated area: $\boldsymbol{a}$ and $\boldsymbol{b}, \boldsymbol{a}$ being the lower, and $\boldsymbol{b}$ being the upper bound as illustrated in Fig. 5. 


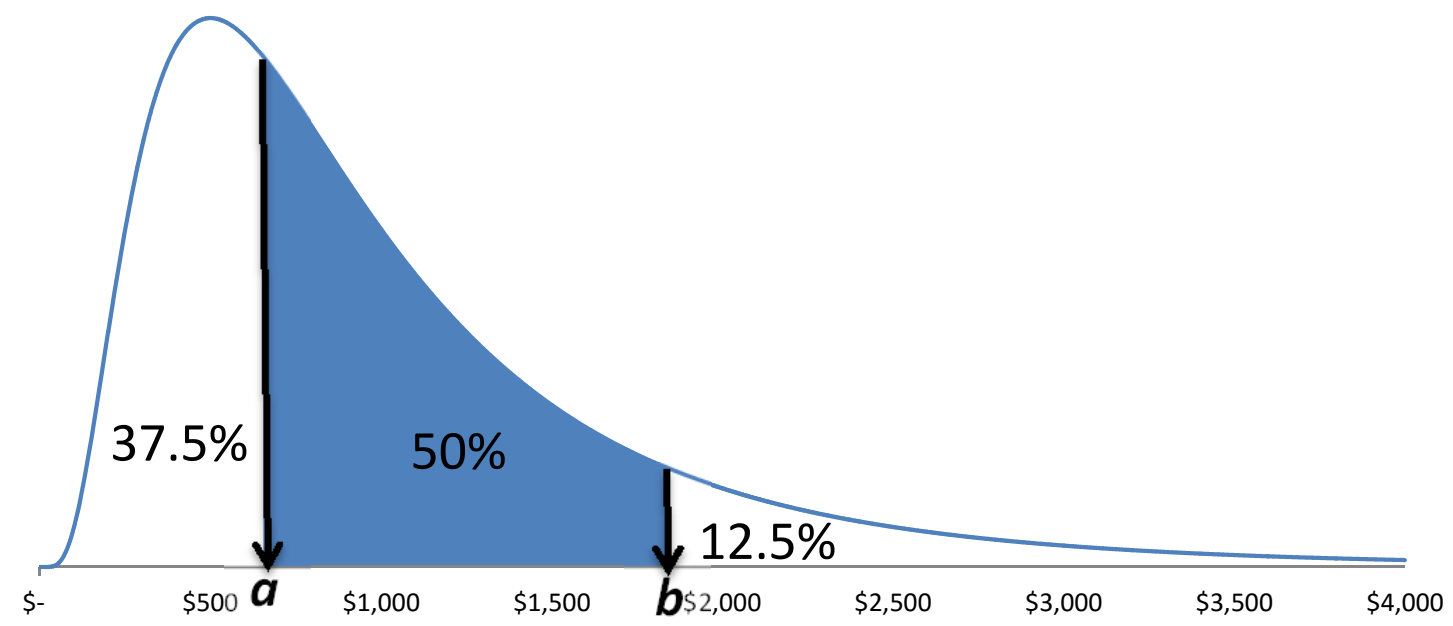

Fig. 5-Illustration for mathematical calculation for directional bias value of 0.5 and overconfidence value of 0.5 . (X-axis in MM\$)

Six steps are required to find the equation of this truncated distribution. First the relative location (right or left) of the investigated area will be calculated based on the directional bias parameter only; optimism will shift the area to the right and pessimism will shift it to the left. Then the exact location of the area will be calculated based on relative location and the overconfidence parameter. The third step is the most involved, and that is calculating the exact values of $\boldsymbol{a}$ and $\boldsymbol{b}$. The fourth step is plugging those values in the truncated distribution equation. The fifth is calculating the expected value of that truncated distribution. The sixth and last step will substitute the mean and standard deviation of the lognormal distribution

The first step is calculating the relative location of the investigated area under the curve. Outside the bounds of this investigated area, the ratio of the area before the point 
$\boldsymbol{a}$ to the area after the point $\boldsymbol{b}$ will be calculated. Those two fractions will add up to one, and will depend only on the directional bias and not the confidence, because we are not calculating the exact percentage of the area yet, only the ratios. The directional bias parameter will be shorthanded to "DB" in this section.

Fraction on the left $=\frac{1+\mathrm{DB}}{2}$.

Fraction on the right $=\frac{1-\mathrm{DB}}{2}$

For example, for a directional bias of 0.5 , the fractions to the left and right are 0.75 and 0.25 , respectively, as shown in Fig. 5.

At this point, we know the ratios and we multiply by the sampled percentage of the area under the curve (confidence) to find the areas at which the truncation should occur. OC will denote the overconfidence parameter in this section.

$$
\begin{aligned}
& \text { Left Area }=\frac{1+\mathrm{DB}}{2}(\mathrm{OC}) \ldots \\
& \text { Right Area }=\frac{1-\mathrm{DB}}{2}(\mathrm{OC}) .
\end{aligned}
$$

For example, for a directional bias of 0.5 and a confidence value of 0.5 , the left area is $37.5 \%$ and the right area is $12.5 \%$, as shown in Fig. 5 .

The next step, and the most involved, is calculating values of the bounds $(\boldsymbol{a}, \boldsymbol{b})$, but this will require solving the inverse equation of the cumulative distribution function of a lognormal distribution. First, I review the probability density function for an unbounded lognormal distribution: 


$$
\log -\mathcal{N}\left(\dot{\mu}, \dot{\sigma}^{2}\right)=\frac{\mathrm{e}^{\frac{-(\ln t-\dot{\mu})^{2}}{2(\dot{\sigma})^{2}}}}{t \dot{\sigma} \sqrt{2 \pi}}, \text { where } t=(-\infty, \infty)
$$

The equation uses $\dot{\mu}$ and $\dot{\sigma}$ because these are not the mean $(\mu)$ and standard deviation $(\sigma)$ that we are interested in when using the Lognormal distribution in this context. A lognormal distribution is defined as the logarithm of a normal distribution, and the mean $\dot{\mu}$ and standard deviation $\dot{\sigma}$ used in this mathematical notation are those of that normal distribution whose logarithm will give the lognormal distribution. Lognormal distribution has a mean of $\mu=e^{\dot{\mu}+\frac{\dot{\sigma}^{2}}{2}}$ and a standard deviation of $\sigma=$ $\sqrt{e^{2 \dot{\mu}+\dot{\sigma}^{2}}\left(e^{\dot{\sigma}^{2}}-1\right)}$, and for simplicity in the notation, we will keep $\dot{\mu}$ and $\dot{\sigma}$ until the last step when we can substitute them for the real mean $\mu$ and standard deviation $\sigma$ by solving the system of those two equations to get equations Eq. 6 and Eq. 7.

$$
\begin{aligned}
& \dot{\mu}=\ln (\mu)-\frac{1}{2} \ln \left(\frac{\mu^{2}+\sigma^{2}}{\mu^{2}}\right) \\
& \dot{\sigma}=\sqrt{\ln \left(\frac{\mu^{2}+\sigma^{2}}{\mu^{2}}\right)} .
\end{aligned}
$$

To find the area under the curve, we need the cumulative distribution function, which is the integration of the probability density function (Eq. 5) from $-\infty$ to $x$.

$$
F_{X}(x ; \dot{\mu}, \dot{\sigma})=\int_{-\infty}^{x} \frac{\mathrm{e}^{\frac{-(\ln t-\mu)^{2}}{2(\dot{\sigma})^{2}}}}{t \dot{\sigma} \sqrt{2 \pi}} d t=\frac{1}{2}\left[1+\operatorname{Erf}\left(\frac{\ln x-\dot{\mu}}{\dot{\sigma} \sqrt{2}}\right)\right] .
$$

Eq. 8 can be used to find the cumulative probability that a certain dollar amount will be sampled from this distribution, for example, the probability that PVOCF will be 
less than 500(\$MM). However, in our case, we do have the cumulative probabilities, $\boldsymbol{a}$ '

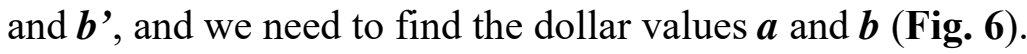

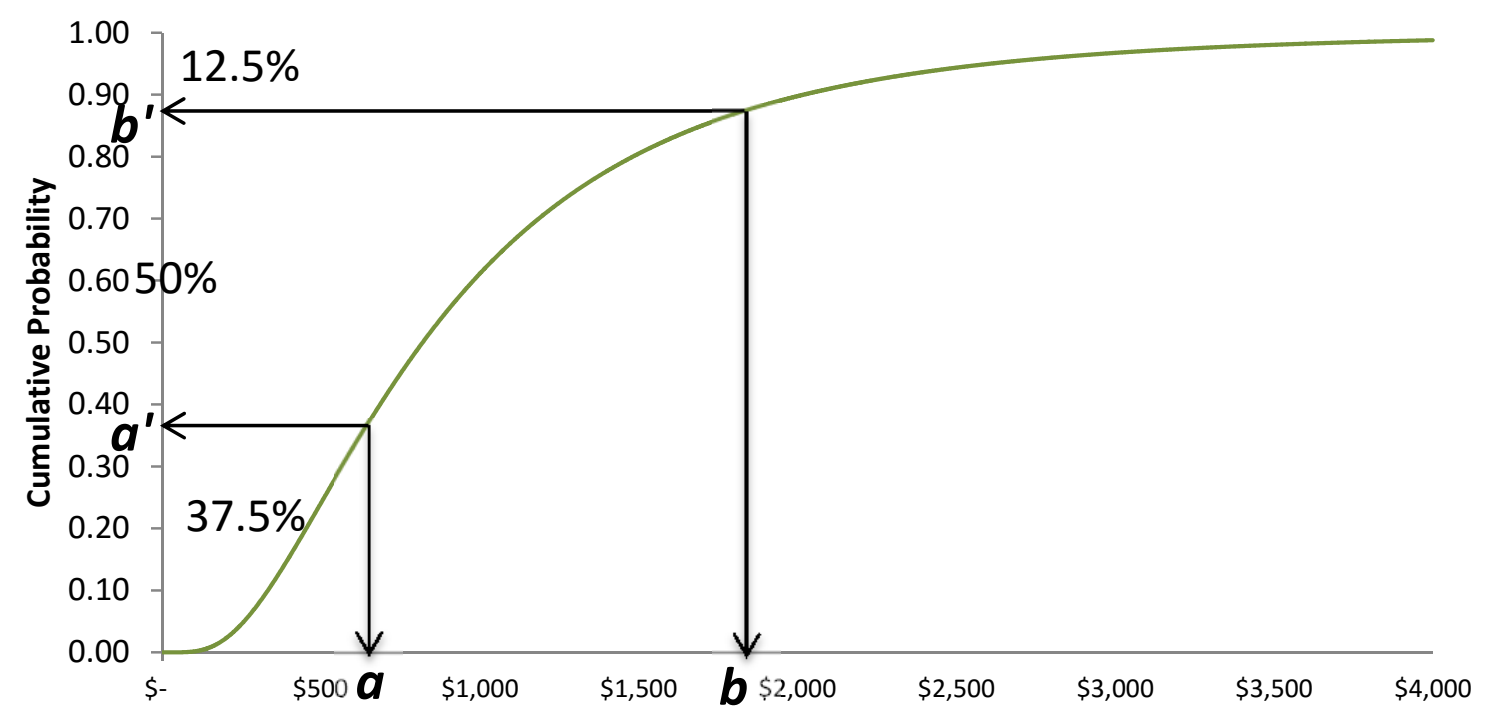

Fig. 6-CDF of PVOCF for overconfidence and directional bias values of 0.5. (Xaxis in MM\$)

Fig. 6 shows the CDF of a typical PVOCF with a 0.5 overconfidence and directional bias, with the $\mathrm{X}$-axis matching that of Fig. 5. The points $\boldsymbol{a}^{\prime}$ and $\boldsymbol{b}$ ' that are marked on the Y-axis are obtained from the left area (Eq. 3), and 1 - right area (Eq. 4). The corresponding points $\boldsymbol{a}$ and $\boldsymbol{b}$ are the ones required as the bounds of the truncated lognormal distribution, and thus an inverse function of the cumulative probability function is required, as follows:

$$
F_{X}^{-1}(q ; \dot{\mu}, \dot{\sigma})=\mathrm{e}^{\dot{\mu}+\dot{\sigma} \sqrt{2} \operatorname{Erf}^{-1}[2 q-1]} .
$$


The lower bound $(\boldsymbol{a})$ is the value at which the area under the curve equals the left area calculated in Eq. 3 and will be substituted in Eq. 9:

$$
a=F_{X}^{-1}(\text { Left Area })=F_{X}^{-1}\left(\frac{1+\mathrm{DB}}{2}(\mathrm{OC})\right)=e^{\dot{\mu}+\dot{\sigma} \sqrt{2} \operatorname{Erf}^{-1}\left[2 \frac{1+\mathrm{DB}}{2}(\mathrm{OC})-1\right]}
$$

The last step in calculating the lower bound $\boldsymbol{a}$ is to substitute the real $\mu$ and $\sigma$ from Eq. 6 and Eq. 7.

$$
a=\frac{\mu^{2} e^{\sqrt{2} \operatorname{Erf}^{-1}[\mathrm{OC}+\mathrm{DB}(\mathrm{OC})-1] \sqrt{\ln \left[\mu^{2}+\sigma^{2}\right]-2 \ln [\mu]}}}{\sqrt{\mu^{2}+\sigma^{2}}} .
$$

For example, using Eq. 10, and knowing that the lognormal distribution in Fig. 5 is a typical PVOCF, lognormal (mean $=\$ 1050 \mathrm{MM}, \mathrm{SD}=\$ 840)$, the value of $\boldsymbol{a}$ can be calculated to be $\$ 655 \mathrm{MM}$ for confidence and directional bias values both equal to 0.5 .

The upper bound $\boldsymbol{b}$ is the quantile at which the remainder of the area under the curve equals the right area calculated in Eq. 4. So (1-right area) will be substituted in Eq. 9.

$b=F_{X}^{-1}(1-$ Right Area $)=F_{X}^{-1}\left(1-\frac{1-\mathrm{DB}}{2}(\mathrm{OC})\right)=e^{\dot{\mu}+\dot{\sigma} \sqrt{2} \operatorname{Erf}^{-1}\left[2\left[1-\frac{1-\mathrm{DB}}{2}(\mathrm{OC})\right]-1\right]}$.

After substituting the real $\mu$ and $\sigma$ from equations Eq. 6 and Eq. 7, we end up with an expression very similar to $\boldsymbol{a}$ in Eq. 10, except for the expression inside the inverse error function.

$$
b=\frac{\mu^{2} e^{\sqrt{2} \operatorname{Erf}^{-1}[1-\mathrm{OC}+\mathrm{DB}(\mathrm{OC})] \sqrt{\ln \left[\mu^{2}+\sigma^{2}\right]-2 \ln [\mu]}}}{\sqrt{\mu^{2}+\sigma^{2}}} .
$$

For example, substituting in Eq. 11 confidence and directional bias values of 0.5 , and lognormal $($ mean $=\$ 1050 \mathrm{MM}, \mathrm{SD}=\$ 840)$ gives a value of $\$ 1,841 \mathrm{MM}$ for $\boldsymbol{b}$. 
After locating the actual bounds $\boldsymbol{a}$ and $\boldsymbol{b}$, the fourth step is to find an equation for the truncated lognormal distribution from $\boldsymbol{a}$ to $\boldsymbol{b}$. First we start with the general equation for truncated lognormal distribution:

$$
\log -\mathcal{N}_{\text {truncated }}\left(a, b ; \dot{\mu}, \dot{\sigma}^{2}\right)=\frac{\log -\mathcal{N}\left(\dot{\mu}, \dot{\sigma}^{2}\right)}{F_{X}\left(b ; \dot{\mu}, \dot{\sigma}^{2}\right)-F_{X}\left(a ; \dot{\mu}, \dot{\sigma}^{2}\right)}
$$

Then, we substitute Eq. 5 in the numerator, and expand Eq. 8 in the denominator for both $\boldsymbol{a}$ and $\boldsymbol{b}$ inside the cumulative distribution functions to give:

$$
\text { Log- } \mathcal{N}_{\text {truncated }}=\frac{\frac{\mathrm{e}^{\frac{-(\ln t-\dot{\mu})^{2}}{2(\dot{\sigma})^{2}}}}{t \dot{\sigma} \sqrt{2 \pi}}}{\frac{1}{2}\left[1+\operatorname{Erf}\left(\frac{\ln b-\dot{\mu}}{\dot{\sigma} \sqrt{2}}\right)\right]-\frac{1}{2}\left[1+\operatorname{Erf}\left(\frac{\ln a-\dot{\mu}}{\dot{\sigma} \sqrt{2}}\right)\right]}
$$

After finding the equation, the fifth step is to find the expected value of the distribution. Shift from the expected value of the original distribution constitutes bias that will be translated to disappointment in the estimation process. The estimated value for a given directional bias and overconfidence is the mean of the truncated lognormal distribution in Eq. 12.

$$
\begin{aligned}
& \text { mean }=\int_{a}^{b} x f(x) d x=\int_{a}^{b} x \times \log -\mathcal{N}_{\text {truncated }}\left(a, b ; \dot{\mu}, \dot{\sigma}^{2}\right) d x \\
& =\int_{a}^{b} x \frac{\frac{\mathrm{e}^{\frac{-(\ln x-\dot{\mu})^{2}}{2(\dot{\sigma})^{2}}}}{x \dot{\sigma} \sqrt{2 \pi}}}{\frac{1}{2}\left[1+\operatorname{Erf}\left(\frac{\ln b-\dot{\mu}}{\dot{\sigma} \sqrt{2}}\right)\right]-\frac{1}{2}\left[1+\operatorname{Erf}\left(\frac{\ln a-\dot{\mu}}{\dot{\sigma} \sqrt{2}}\right)\right]} \mathrm{dx} \\
& E V=\frac{\mathrm{e}^{\dot{\mu}+\frac{(\dot{\sigma})^{2}}{2}}\left[\operatorname{Erf}\left(\frac{\dot{\mu}+(\dot{\sigma})^{2}-\ln a}{\dot{\sigma} \sqrt{2}}\right)-\operatorname{Erf}\left(\frac{\dot{\mu}+(\dot{\sigma})^{2}-\ln b}{\dot{\sigma} \sqrt{2}}\right)\right]}{\operatorname{Erf}\left(\frac{\ln b-\dot{\mu}}{\dot{\sigma} \sqrt{2}}\right)-\operatorname{Erf}\left(\frac{\ln a-\dot{\mu}}{\dot{\sigma} \sqrt{2}}\right)}
\end{aligned}
$$


The sixth step involves substituting the values of the real $\mu$ and $\sigma$ from equations Eq. 6 and Eq. 7.

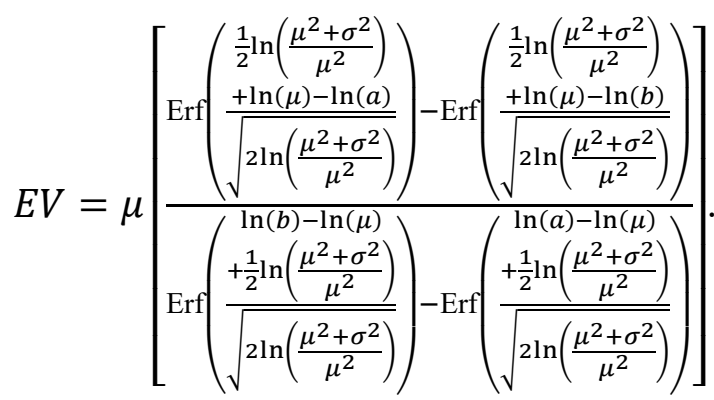

The equations of $\boldsymbol{a}$ and $\boldsymbol{b}$ can also be substituted from Eq. 10 and Eq. 11 into Eq. 14 to give Eq. 15 for completeness, but that can be easily avoided numerically by preevaluating $\boldsymbol{a}$ and $\boldsymbol{b}$ in a sequential fashion. 


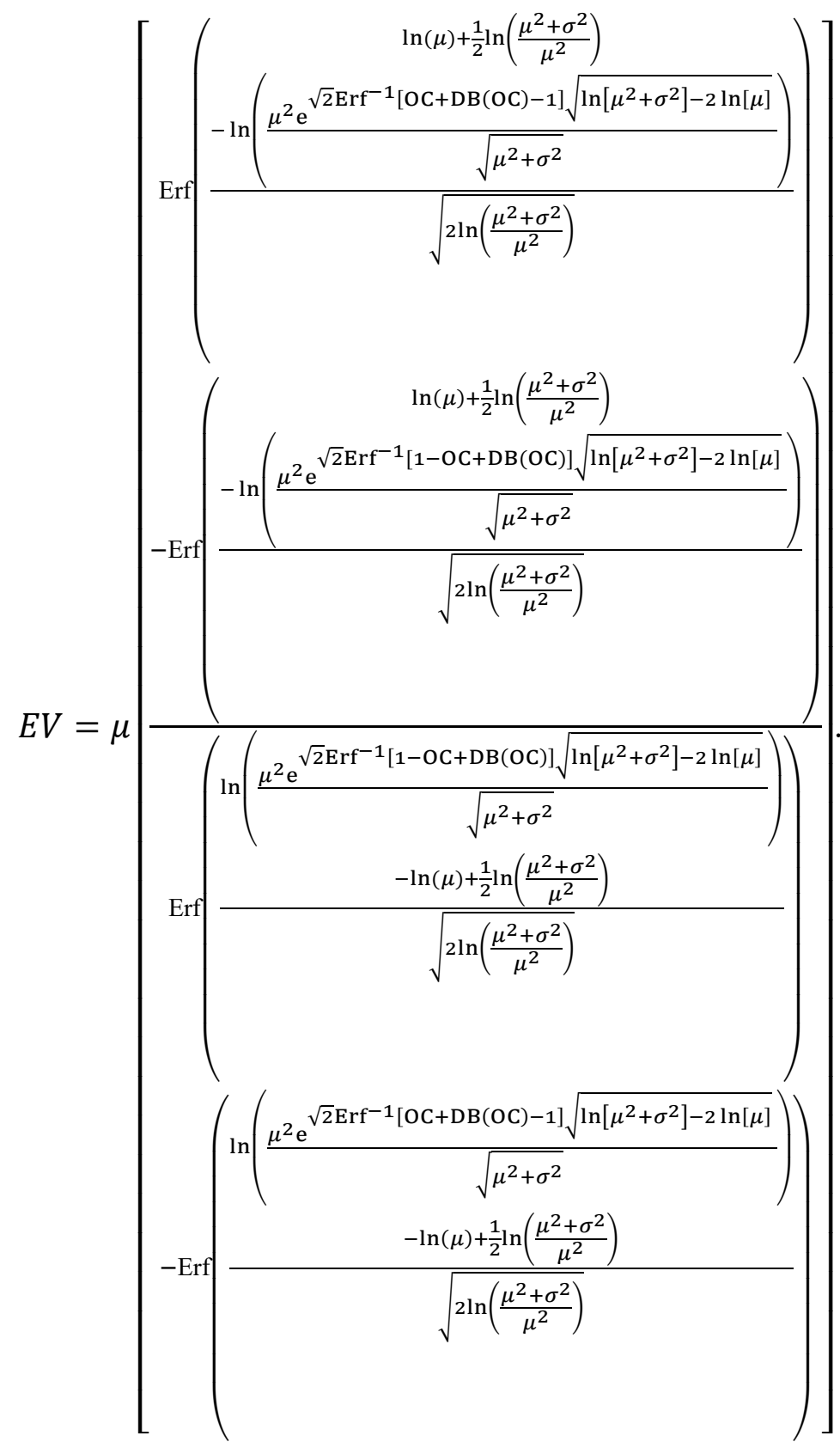

Implementation

The first step in the Monte-Carlo simulation of the new framework is the implementation of the equations that links the true EV's with the estimated EV's. Table 3 outlines the 
steps involved in generating the true EV's of a project, and the implementation of relevant equations to generate the estimated EV's, given certain values for confidence and directional bias. Those steps will be repeated one hundred times to generate 100 projects that constitute the global portfolio that is available to one company or estimator.

The table shows each intermediate parameter in a specific row, the description of that parameter, and then a sample value of that parameter based on the underlying distribution and calculations.

Table 3-Implementation of calculations

\begin{tabular}{|c|c|c|c|c|}
\hline \multirow{3}{*}{$\begin{array}{l}1 \\
2\end{array}$} & Type & Name & Description & Sample value \\
\hline & \multirow{5}{*}{ 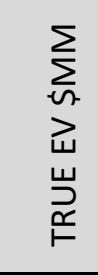 } & PVOCF & Present Value of Operating Cash & $\$ 1,050$ \\
\hline & & CapEx & Capital Expenditure & 700 \\
\hline 3 & & NPV & Net Present & 350 \\
\hline 4 & & IE & Investment Efficiency & 0.5 \\
\hline 5 & & Rank & True IE rank of the project & 1 \\
\hline 6 & \multirow{2}{*}{ 崖 } & PVOCF & Standard deviation of the PVOCF (Random * row1) & 840 \\
\hline 7 & & CapEx & Standard deviation of the CapEx (Random * row2) & $\$ 560$ \\
\hline 8 & \multirow{2}{*}{ ฉี } & PVOCF & Directional bias for the PVOCF & 0.50 \\
\hline 9 & & CapEx & Directional bias for the CapEx & -0.50 \\
\hline 10 & \multirow{2}{*}{ ○ } & PVOCF & Overconfidence parameter for the PVOCF & \multirow{4}{*}{$\begin{array}{r}0.50 \\
0.50 \\
\$ \quad 654 \\
\$ 1,836\end{array}$} \\
\hline 11 & & CapEx & Overconfidence parameter of the CapEx & \\
\hline 12 & \multirow{9}{*}{ 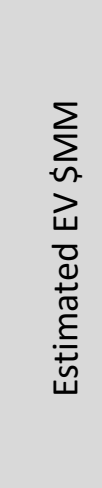 } & \multirow{3}{*}{ PVOCF } & a: Minimum of the investigated range of PVOCF & \\
\hline 13 & & & b: Maximum of the investigated range of PVOCF & \\
\hline 14 & & & The expected value of the estimated PVOCF & $\$ 1,088$ \\
\hline 15 & & \multirow{3}{*}{ CapEx } & a: Minimum of the estimated range of CapEx & 244 \\
\hline 16 & & & b: Maximum of the estimated range of CapEx & $\$ 685$ \\
\hline 17 & & & The expected value of the estimated CapEx & 446 \\
\hline 18 & & NPV & Estimated NPV of the project & 642 \\
\hline 19 & & IE & Estimated Investment Efficiency & 1.437392 \\
\hline 20 & & Rank & Estimated IE rank of this project & 1 \\
\hline
\end{tabular}


The implementation will require 20 parts divided into five segments, as outlined in Table 3. The segments calculate the true EV, standard deviation of true EV, directional bias, overconfidence, and estimated EV.

In the first segment, the true EV is calculated. First, PVOCF value for the project is randomly sampled from the lognormal distribution mean $=\$ 750 \mathrm{MM}, \mathrm{SD}=\$ 750 \mathrm{MM}$, shift $=\$ 300 M M$. Second, the CapEx value is independently sampled from Begg and Bratvold lognormal distribution mean $=\$ 600 \mathrm{MM}, \mathrm{SD}=\$ 600 \mathrm{MM}$, shift $=\$ 100 \mathrm{MM}$. Then, the NPV, IE, and rank are calculated. NPV $=$ PVOCF - CapEx, IE $=$ NPV/CapEx, and the rank of this project within the set of 100 randomly generated projects can be calculated by sorting the projects based on the IE that was just calculated.

The second segment calculates the standard deviations of the true EVs. Standard deviation of the PVOCF is randomly sampled from a Pert distribution min $=0.3$, mode $=0.8, \max =1.3$, and then that random factor is multiplied by the PVOCF EV calculated earlier in row number 1 . The second standard deviation is that of the CapEx and is randomly sampled from a Pert distribution $\min =0.3$, mode $=0.8, \max =1.3$, and then multiplied by the CapEx EV calculated earlier in row number 2.

The third segment will specify the directional bias. This value will be varied manually to probe the effect of directional bias on the estimation process. Two modes of calculating the directional bias are used. The first is deterministic mode, where the fixed value of directional bias is used as specified. The second is stochastic mode, where the specified value will seed a small normal distribution, with a standard deviation of 0.2 , to find a final directional bias that varies from project to project. The first item in this 
segment is the directional bias for the PVOCF, and it will be set to the desired directional bias value assigned to PVOCF in the deterministic biases case. The stochastic case will use a small normal distribution with a mean equal to the assigned value and a standard deviation of 0.2 . The second item in this segment is the directional bias for the CapEx and it will be calculated in a similar way but in the opposite direction (assigned value will be negated) because CapEx is a cost parameter.

The forth segment will calculate the overconfidence parameter. This value too will be varied manually to study the effect of overconfidence on the estimation process. Similarly, deterministic and stochastic modes are calculated in the same way the directional bias is calculated.

The fifth and last segment will calculate the estimated value based on all the previous parameters. As outlined from the mathematical formulation, pre-calculating the endpoints in the range of the estimated distribution is simpler in the implementation than doing all the calculation in one step. Starting with the PVOCF, the $a$ parameter, which is the minimum of the investigated range of PVOCF, will be calculated based on Eq. 10 using the mean (row 1), standard deviation (row 6), directional bias (row 8), and overconfidence (row 10). The $\boldsymbol{b}$ parameter, which is the maximum of the investigated range of PVOCF, will be calculated using Eq. 11 and using the same parameters as for the row above. The next step is calculating the expected value of the estimated PVOCF, which is calculated using $\boldsymbol{a}$ and $\boldsymbol{b}$ from rows 12, and 13 above, and using Eq. 14. The same technique will be used with CapEx. The range of the estimated distribution will be calculated first, and then the expected value of the estimated distribution. The minimum 
of the estimated range of CapEx, a, will be calculated based on Eq. 10, but using the mean of the CapEx (row 2), standard deviation of the CapEx (row 7), directional bias of the CapEx (row 9), and overconfidence of CapEx (row 11). The maximum of the estimated range of CapEx, $\boldsymbol{b}$, will be calculated using Eq. 11 and using the same parameters as the row above. The expected value of the estimated CapEx will be calculated using $\boldsymbol{a}$ and $\boldsymbol{b}$ from rows 15, and 16 above, and using Eq. 14. The estimated NPV is easy to calculate now by subtraction: PVOCF (row 14) - CapEx (row 17). The investment efficiency (IE) is then calculated from the NPV and the CapEx: NPV (row 18) / CapEx (row 17). Now, the rank of this project within the 100 available projects is calculated by sorting them by the estimated IE.

\section{Unconstrained Budget Calculations}

This evaluation method assumes budget is available to fund all projects and chooses to execute all available projects that have a positive NPV. The first level of analysis is reviewing the 100 projects by examining their true NPV and estimated NPV, rows 3 and 18 of Table 3, respectively, to determine if each project is correctly accepted, correctly rejected, wrongfully accepted, or wrongfully rejected. Accepted projects are those that have positive NPV, and rejected projects are those that have negative NPV. Correctly accepted projects are those that have positive true NPV and positive estimated NPV. Correctly rejected projects have negative true NPV and negative estimated NPV. Wrongfully accepted projects are those that are accepted because they have positive estimated NPV but they should not have been selected because they have a negative true 
NPV. Wrongfully rejected projects are those that are rejected because of negative estimated NPV but should have been selected because they have positive true NPV.

The next step is to accumulate those results (Table 4). The numbers in Table 4 change with each random iteration; the numbers recorded in Table 4 correspond to the average results for an overconfidence value of 0.5 and a directional bias of 0.5 . The table starts with listing the number of projects that were selected or rejected based on the estimated NPV, vertically, and then by the true NPV, horizontally. For example, the table shows that 88 projects were selected based on positive estimated NPV and 73 of them had a positive true NPV (correctly selected), but 15 did not (wrongfully selected). Likewise, 12 projects were rejected based on their negative estimated NPV and they all were correctly rejected ( 0 wrongfully rejected). Then, the estimated NPV's for those selected projects in each category are summed in the next part of the table, and the last part shows the sums of the true NPV's for the same set of projects. The format of this table is similar to the Begg and Bratvold (2008) analysis. 
Table 4-Analysis of unconstrained budget estimation results

\begin{tabular}{|c|c|c|c|c|}
\hline \multirow{3}{*}{\multicolumn{2}{|c|}{$\begin{array}{c}\text { Number of } \\
\text { projects }\end{array}$}} & \multirow{2}{*}{\multicolumn{2}{|c|}{ Estimates }} & \multirow[b]{3}{*}{ Total } \\
\hline & & & & \\
\hline & & Rejected & & \\
\hline \multirow{2}{*}{ TRUE } & Rejected & & 15 & 27 \\
\hline & Accepted & & 73 & 73 \\
\hline Total & & & 88 & 100 \\
\hline
\end{tabular}

\begin{tabular}{|c|c|c|c|c|}
\hline \multirow{2}{*}{\multicolumn{2}{|c|}{ Estimated NPV }} & \multicolumn{2}{|c|}{ Estimates } & \multirow[b]{2}{*}{ Total } \\
\hline & & Rejected & Accepted & \\
\hline \multirow{2}{*}{ TRUE } & Rejected & $\$(5,905.52)$ & $\$ \quad 3,414.70$ & $\$(2,490.81)$ \\
\hline & Accepted & $\$$ & $\$ 61,770.56$ & $\$ 61,770.56$ \\
\hline Total & & $\$(5,905.52)$ & $\$ 65,185.27$ & $\$ 59,279.75$ \\
\hline
\end{tabular}

\begin{tabular}{|c|c|c|c|c|}
\hline & & & & \multirow[b]{3}{*}{ Total } \\
\hline \multirow{2}{*}{\multicolumn{2}{|c|}{ True NPV }} & \multicolumn{2}{|c|}{ Estimates } & \\
\hline & & Rejected & Accepted & \\
\hline \multirow{2}{*}{ TRUE } & Rejected & $\$(12,968.42)$ & $\$(3,303.31)$ & $\$(16,271.74)$ \\
\hline & Accepted & $\$$ & $\$ 48,596.63$ & $\$ 48,596.63$ \\
\hline Total & & $\$(12,968.42)$ & $\$ 45,293.32$ & $\$ 32,324.90$ \\
\hline
\end{tabular}

The last step of this analysis is to calculate the output parameters. First, the Expected Disappointment as a percent of estimated NPV $(\mathrm{ED} \% \mathrm{E})$ is the difference between the estimated and realized NPV of the portfolio normalized by the estimated NPV (Eq. 16). The Estimated NPV (EstNPV) of the portfolio is the total of estimated NPV's of accepted projects based on their estimated NPV ( 88 projects), and that value is $\$ 65,185.27$ in the second part of the table as the total under the accepted based on estimates. The realized NPV (RNPV) of the portfolio is the total of the true NPV's of the projects that were selected based on their positive estimated NPV's (the same 88 projects), and that value is $\$ 45,293.32$ in the third part of the table as the total under the accepted based on estimates. "Realized" refers to the resultant value of the portfolio after 
execution of the projects. For the numbers shown in Table 4, the instantaneous value of disappointment as a percentage of estimated NPV would be: $\frac{65,185.27-45,293.32}{65,185.27}=30.5 \%$. This instantaneous result will be accumulated over all Monte-Carlo iterations and the average of all the numbers is the expected value of disappointment as a percentage of estimated NPV (ED\%E).

$$
E D \% E=E\left(\frac{E s t N P V-R N P V}{E s t N P V}\right)
$$

The second output parameter is the Expected Decision Error as a percentage of the estimated NPV $(\mathrm{EDE} \% \mathrm{E})$, which is the difference between the realized NPV (RNPV) and the Best Possible NPV (BPNPV) normalized by the estimated NPV (Eq. 17). The BPNPV portfolio is the highest value portfolio that could be selected from this set of projects, and can be found by choosing all the projects with positive true NPV from the set (73 in this case). The BPNPV is $\$ 48,596.63$, the total of the true accepted projects (across) in the third part of the table. For the example in Table 4, the decision error as a percentage of estimated NPV would be: $\frac{48,596.63-45,293.32}{65,185.27}=5.07 \%$.

$$
E D E \% E=E\left(\frac{B P N P V-R N P V}{E s t N P V}\right)
$$

To find the expected value of the decision error, the values from multiple MonteCarlo iterations are accumulated and the average number reported.

\section{Constrained Budget Calculations}

The main difference between constrained and unconstrained budget calculations is that the constrained budget calculations takes into account the available budget in the 
decision making. Most of the calculation logic follows Begg and Bratvold (2008) and assumptions were made when they have not clearly specified the calculation method. The calculations go through three different analysis phases—-for the best possible scenario, the estimated scenario, and the realized scenario-and then one last step to collect the output of those three phases.

The first analysis phase is to account for the best possible scenario. The 100 projects are sorted based on their true IE (row 4 from Table 3). Then, the number of projects with a positive true NPV (row 3 from Table 3 ) will be noted so that the rest of the projects will be excluded from the calculations. Then, starting with the highest true IE projects, the number of projects whose combined true CapEx will fit within the assigned budget is determined and their true NPV's are summed to determine the Best Possible NPV (BPNPV). After that, a partial percentage of the true CapEx of the next project from the top of the list is added to make the budget fully utilized, and the same percentage of its true NPV is added to the total BPNPV.

The second analysis phase is to account for the estimated scenario. The list of 100 projects is sorted again, but this time according to their estimated IE (row 19 from Table 3). Again, the projects with negative estimated NPV's (row 18 in Table 3) are excluded from the calculations. The combined estimated CapEx (row 17 from Table 3) of the top projects are compared against the budget and the estimated NPV's of those projects that fit within the budget will be summed to get the Estimated NPV (EstNPV). The last step is to add a percentage of the estimated CapEx of the next project on the list 
to fill in the remaining budget, and the same percentage of its estimated NPV is added to the EstNPV.

The last analysis phase accounts for the realized (or actual) scenario, where projects are selected based on their estimated values but once the company commits to those projects, the true values will be realized. The list of projects are sorted according to their estimated IE's, as in the estimation phase. The negative projects are excluded from the calculations based on the estimated NPV. However, this time the number of projects executed is based on the true CapEx. The assumption here is that as the projects are executed, the true CapEx will be revealed, and the company will either decrease the number of projects chosen in the estimation phase not to overcommit itself in the optimistic cases, or will add more projects to fill up the remaining budget in the pessimistic cases. However, projects with negative estimated NPV will never be added to fill up the budget because they were excluded from the previous step. This will affect the pessimistic scenario the most, where the estimation will amplify the CapEx values and chose fewer projects than potentially possible. When the projects start and true CapEx is revealed, they will find they have extra money to spare, but will not attempt to invest in projects they deemed losing projects $(\mathrm{EstNPV}<0)$ from the beginning. This could create scenarios where not all the budget is invested, and in those scenarios, the rest of the budget is added to NPV, as cash. Once the number of projects that will fit the budget is settled, the true NPV's of those projects will be summed for the realized NPV (RNPV). A percentage of true CapEx of the next project on the list is added to the 
budget, provided that such a project had a positive estimated NPV, and then the same percentage of the true NPV of that project is added to the RNPV.

The last step is to calculate the $\mathrm{ED} \% \mathrm{E}$ and $\mathrm{EDE} \% \mathrm{E}$, using the same equations used in the unconstrained budget case, Eq. 16 and Eq. 17. 


\section{RESULTS*}

\section{Impact with Deterministic Biases}

In this section the impact of biases using fixed values of the confidence and directional bias parameters will be assessed. In particular, expected disappointment and expected decision error for fixed confidence parameter values ranging from 0.0 (zero overconfidence) to 1.0 (deterministic) and fixed directional bias parameter values ranging from -1.0 (complete pessimism) to 1.0 (complete optimism) will be calculated.

\section{Unconstrained-budget case}

We first consider expected disappointment (Fig. 7) for the unconstrained-budget case and see that, with zero overconfidence, $\mathrm{ED} \% \mathrm{E}$ is zero regardless of the degree of directional bias along the $\mathrm{x}$-axis. With greater overconfidence values and zero directional bias along the $\mathrm{y}$-axis, $\mathrm{ED} \% \mathrm{E}$ is not zero as might be expected. $\mathrm{ED} \% \mathrm{E}$ is actually negative, indicating pleasant post-decision surprise. This is a side-effect from the use of lognormal distributions for CapEx and PVOCF. Truncating the same amount of probability from each end of the distribution (overconfidence with zero directional bias) does not preserve the mean of a lognormal distribution. Thus, estimated expected values are different from true expected values, which results in suboptimal project selections and disappointment or pleasant surprise. While the result for overconfidence

\footnotetext{
* Reproduced with permission of Society of Petroleum Engineers (SPE) to reuse SPE-160189 "The Value of Assessing Uncertainty," doi:10.2118/160189-PA. Copyright 2014, Society of Petroleum Engineers Inc. Further reproduction prohibited without permission.
} 
with zero directional bias is small negative disappointment for the particular set of global portfolio parameters that is used, the possibility that overconfidence with zero directional bias could result in small positive ED\%E for a different set of global portfolio parameters cannot be ruled out.

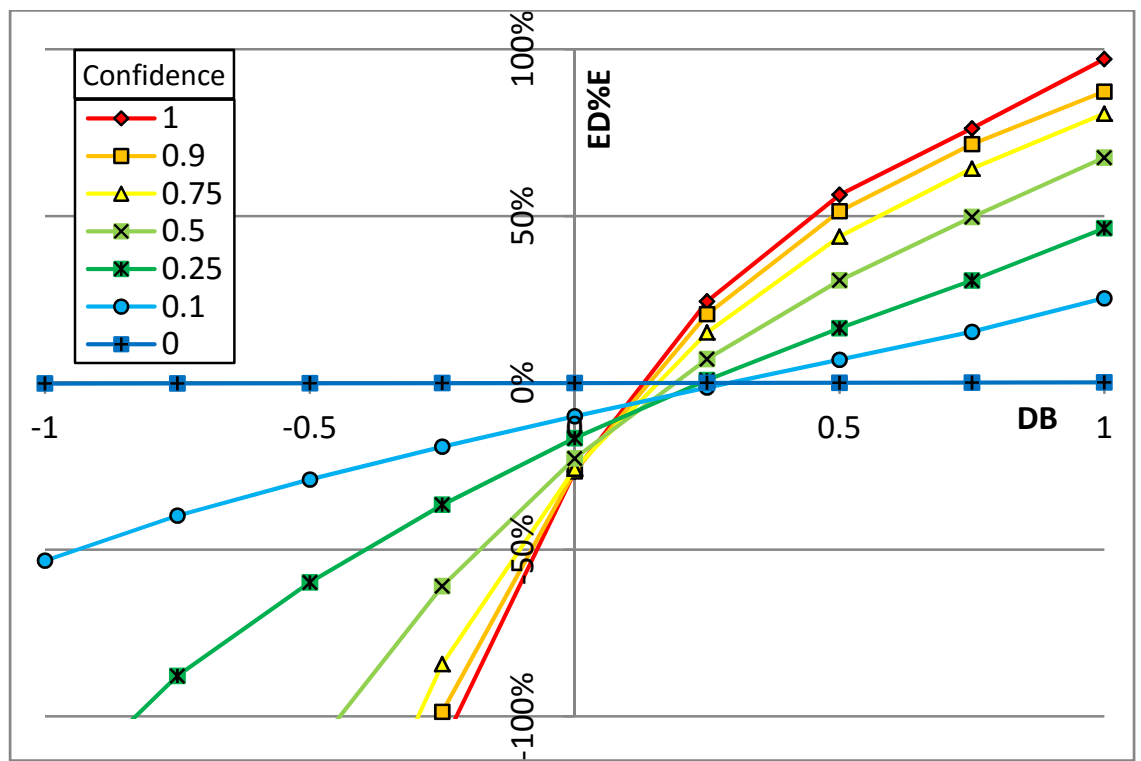

Fig. 7-Expected disappointment, unconstrained budget with deterministic bias.

With nonzero values of directional bias, $\mathrm{ED} \% \mathrm{E}$ increases monotonically as the value of directional bias increases (increasing optimism). In general, as the absolute value of the directional bias increases, the error in estimated EV increases, and this is consistent with the simple illustration in Fig. 1, resulting in increasing magnitude of $\mathrm{ED} \% \mathrm{E}$. With negative directional bias (pessimism), the disappointment is negative (pleasant post-decision surprise). 
An increase in the magnitude of the confidence parameter (increasing overconfidence) results in greater $\mathrm{ED} \% \mathrm{E}$ right of a crossover point at about $0.15-0.2$ directional bias and results in greater pleasant surprise left of this crossover point. Increasing overconfidence magnifies the error in estimated $\mathrm{EV}$, again consistent with Fig. 1, which increases the magnitude of $\mathrm{ED} \% \mathrm{E}$.

The magnitude of ED\%E with the addition of directional bias can be large. With confidence equal to 0.9 (high overconfidence), ED\%E ranges from around $-200 \%$ with moderate pessimism to about $90 \%$ with complete optimism. ED\%E of $100 \%$ corresponds to a realized NPV of zero. With moderate values of overconfidence $($ confidence $=0.5)$ and optimism $($ directional bias $=0.5)$, expected disappointment is about $31 \%$. This is significantly higher than the ED values of 5-6\% reported by Begg and Bratvold (2008) attributed to the Optimizer's Curse.

The expected decision error as a percentage of the estimated NPV (EDE\%E) is significantly smaller than percentage expected disappointment $(\mathrm{ED} \% \mathrm{E})$ for the unconstrained-budget case for most values of confidence and directional bias (Fig. 8). With zero directional bias, $\mathrm{EDE} \% \mathrm{E}$ is near zero. With optimism, $\mathrm{EDE} \% \mathrm{E}$ does not exceed $10 \%$, even though $\mathrm{ED} \% \mathrm{E}$ approaches $100 \%$ as directional bias goes to 1 . This indicates that most of the disappointment results from estimation error rather than decision error. 


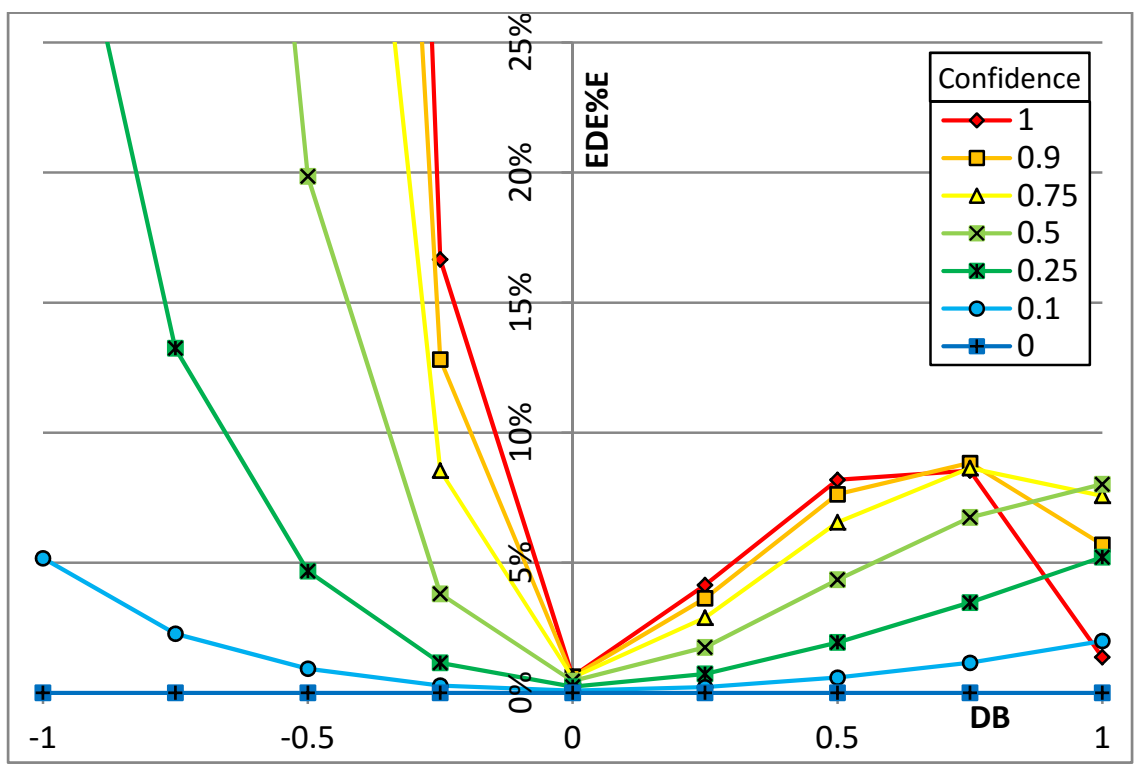

Fig. 8-Expected decision error, unconstrained budget with deterministic bias.

$\mathrm{EDE} \% \mathrm{E}$ increases significantly with increasing pessimism below a directional bias of zero. This happens in part because the estimated NPV decreases with increasing pessimism, but more so because the EDE increases significantly. While some evaluators might find some pessimism to be attractive because it results in pleasant surprise rather than disappointment (Fig. 7), Fig. 8 indicates that the cost of this pleasant surprise is increased decision error; i.e., suboptimal projects are being selected.

\section{Constrained-budget case}

ED\%E for the constrained-budget case (Fig. 9) is similar in character to ED\%E for the unconstrained case (Fig. 7); ED\%E values are typically $75-80 \%$ of the ED\%E values of the unconstrained-budget case for most values of confidence and directional bias. 


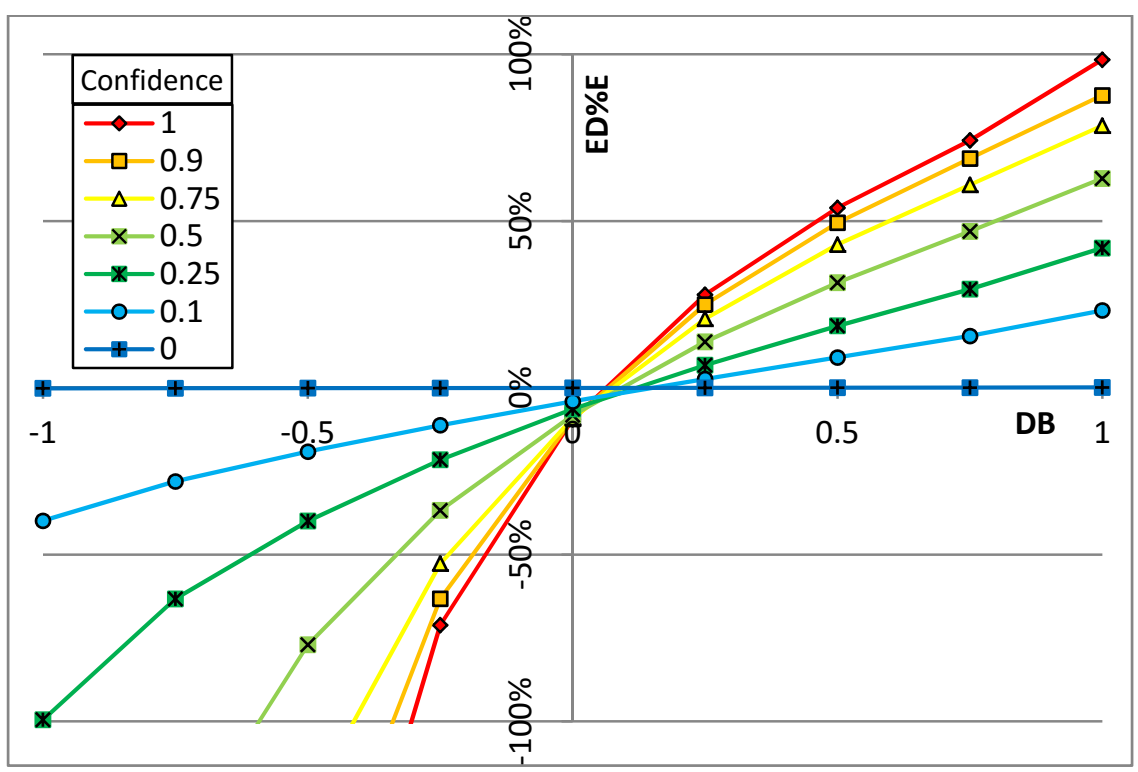

Fig. 9-Expected disappointment, constrained budget with deterministic bias.

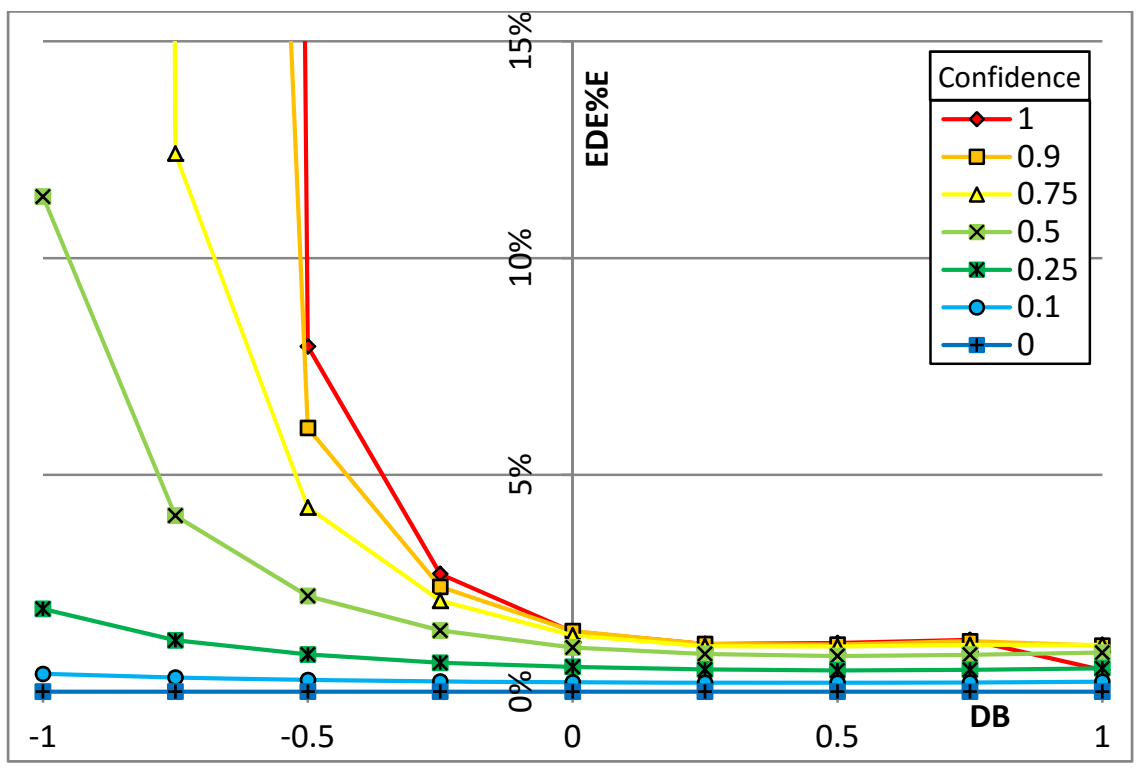

Fig. 10 - Expected decision error, constrained budget with deterministic bias. 
The constrained-budget $\mathrm{ED} \% \mathrm{E}$ results are more realistic because it is more likely that budgets will be constrained in practice. With moderate overconfidence (confidence $=0.5)$ and optimism (directional bias $=0.5$ ), expected disappointment is about $31 \%$. $\mathrm{EDE} \% \mathrm{E}$ for the constrained-budget case is less than $2 \%$ for directional bias greater than or equal to zero (Fig. 10). Below directional bias of zero, EDE\%E increases rapidly with increasing pessimism, although not as rapidly as the unconstrained-budget case.

\section{Impact with Stochastic Biases}

In this section, random variables instead of fixed values for the confidence and directional bias parameters will be used. This is to account for the fact that assessments will not be entirely consistent from project to project within the same company, because

different personnel will be conducting the evaluations. Even if the same person did all of the evaluations using similar methodology, there would still be variability in the degree of bias introduced from project to project. Introducing randomness in the confidence and directional bias parameters introduces randomness in the limited-resources estimated distributions (Fig. 1) and, thus, the estimated EVs that are used in project selection.

The same ranges of confidence and directional bias used in the deterministic bias cases will be run again. However, instead of using fixed values for bias, normal distributions for the bias parameters are used. The normal distributions will have a standard deviation of 0.2 and will be truncated at the appropriate limits for the bias parameters: 0 and 1 for the confidence parameter and -1 and 1 for the directional bias parameter. For example, for a stochastic bias case with overconfidence of 0.5 and 
directional bias of 0.75 , the random variable used for confidence is normal(mean $=0.5$, $\mathrm{SD}=0.2$, truncated at 0 and 1 ) instead of a fixed value of 0.5 , and the random variable used for directional bias is normal(mean $=0.75, \mathrm{SD}=0.2$, truncated at -1 and 1$)$ instead of a fixed value of 0.75 . The values of confidence and directional bias indicated in results plots (Figs. 11-14) corresponds to the means of the distributions used for these parameters.

Overall, ED\%E and EDE\%E for the stochastic bias cases (Figs. 11-14) are similar in character and magnitude to $\mathrm{ED} \% \mathrm{E}$ and $\mathrm{EDE} \% \mathrm{E}$ for the fixed bias cases (Figs. 7-10) for both unconstrained and constrained budget. ED\%E increases from negative to positive as directional bias moves from pessimism to optimism. $\mathrm{EDE} \% \mathrm{E}$ is always positive and significantly smaller than $\mathrm{ED} \% \mathrm{E}$. The stochastic bias cases for overconfidence equal to zero do not yield zero $\mathrm{ED} \% \mathrm{E}$ and $\mathrm{EDE} \% \mathrm{E}$, but this is only because the truncated confidence distribution (normal with mean zero, SD of 0.2 , and truncated at 0 and 1) does not have a mean of zero.

Of particular significance is the large expected disappointment that occurs with moderate overconfidence and optimism, which is likely in practice (Begg et al., 2003; Rose, 2004). With mean confidence $=0.5$ and mean directional bias $=0.5, \mathrm{ED} \% \mathrm{E}$ is $33 \%$ and $36 \%$ for the unconstrained-budget and constrained-budget cases, respectively. These ED\%E values are large enough that they could have significant impact on company financial performance. For example, with these values, portfolios with estimated NPV of $\$ 1$ billion would have actual NPVs of about $\$ 650$ million, on average. 
Greater amounts of either overconfidence and/or optimism would result in lower actual NPVs and greater disappointment.

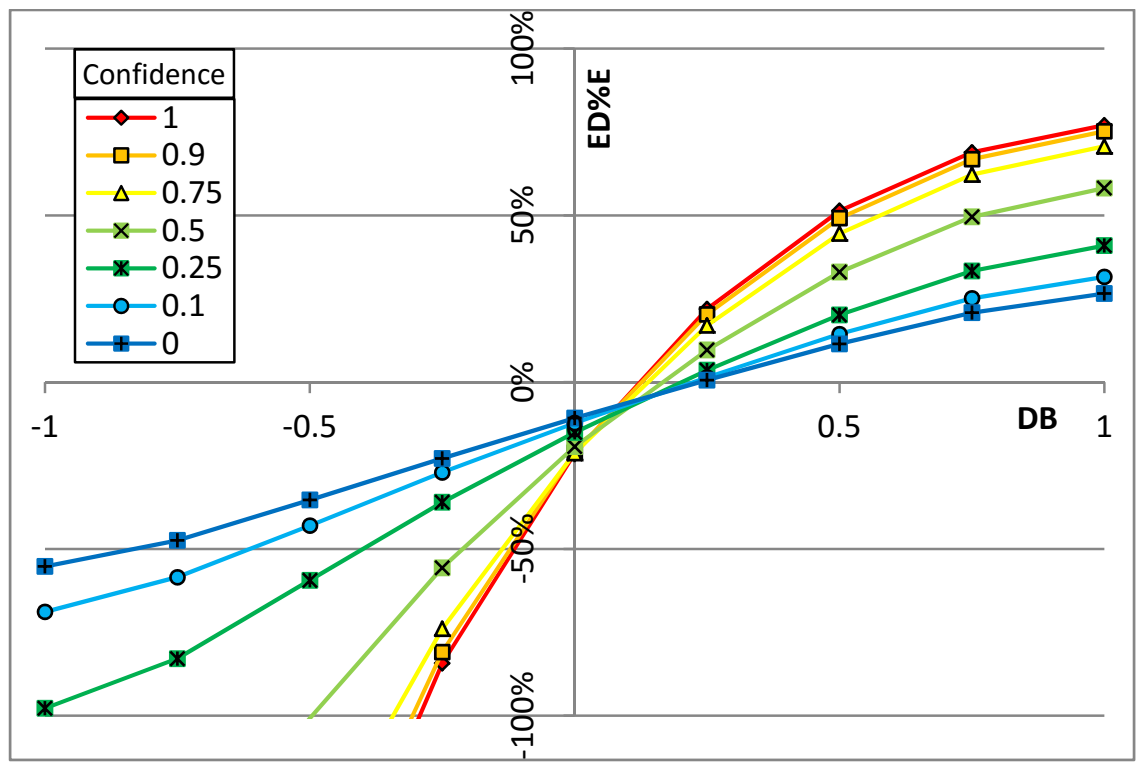

Fig. 11-Expected disappointment, unconstrained budget with stochastic bias. 


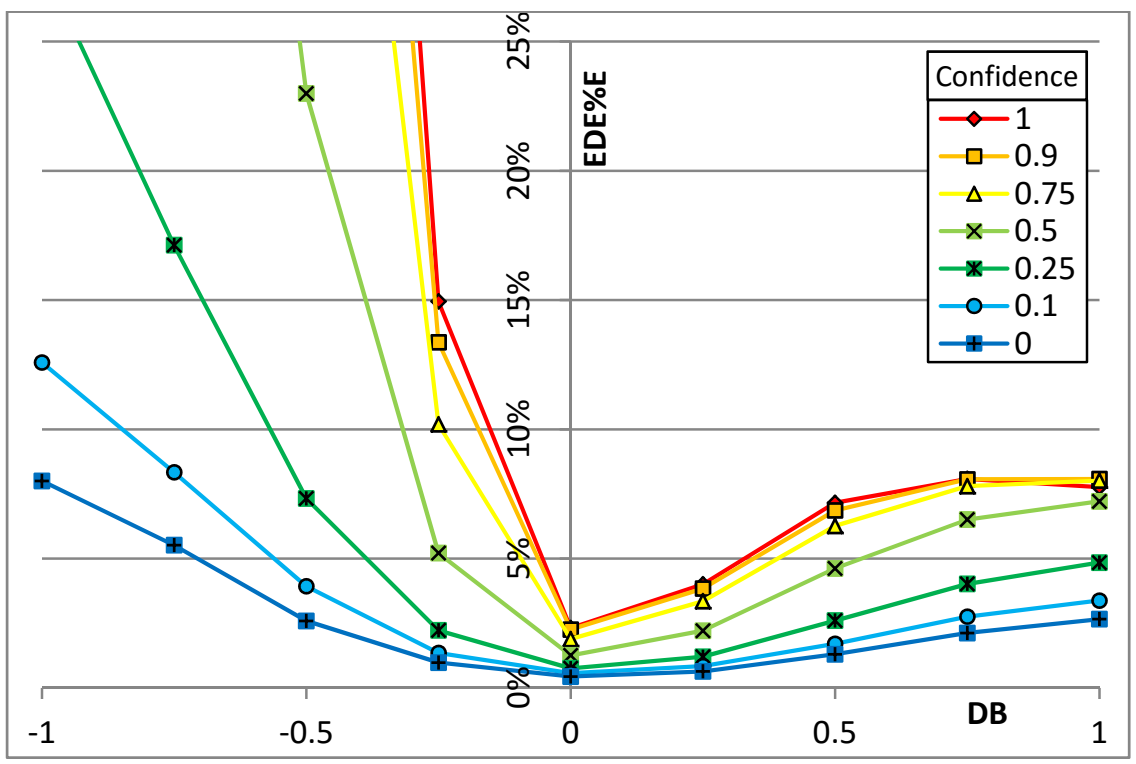

Fig. 12 - Expected decision error, unconstrained budget with stochastic bias.

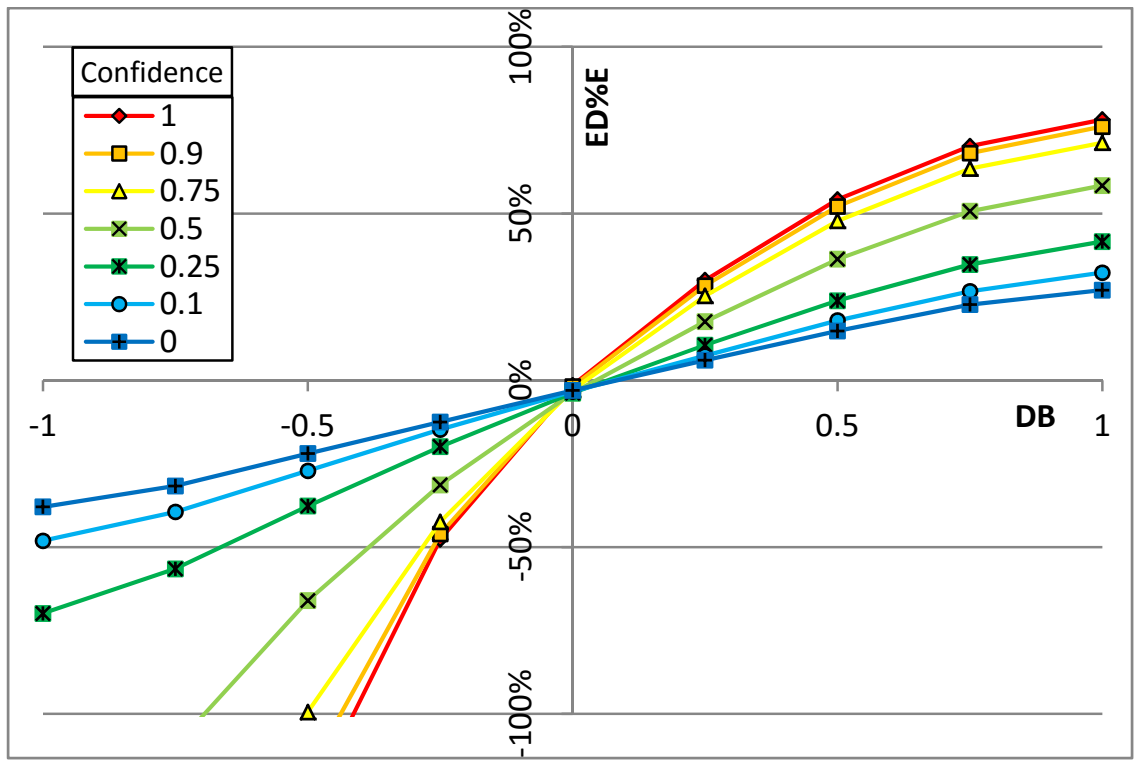

Fig. 13 - Expected disappointment, constrained budget with stochastic bias. 


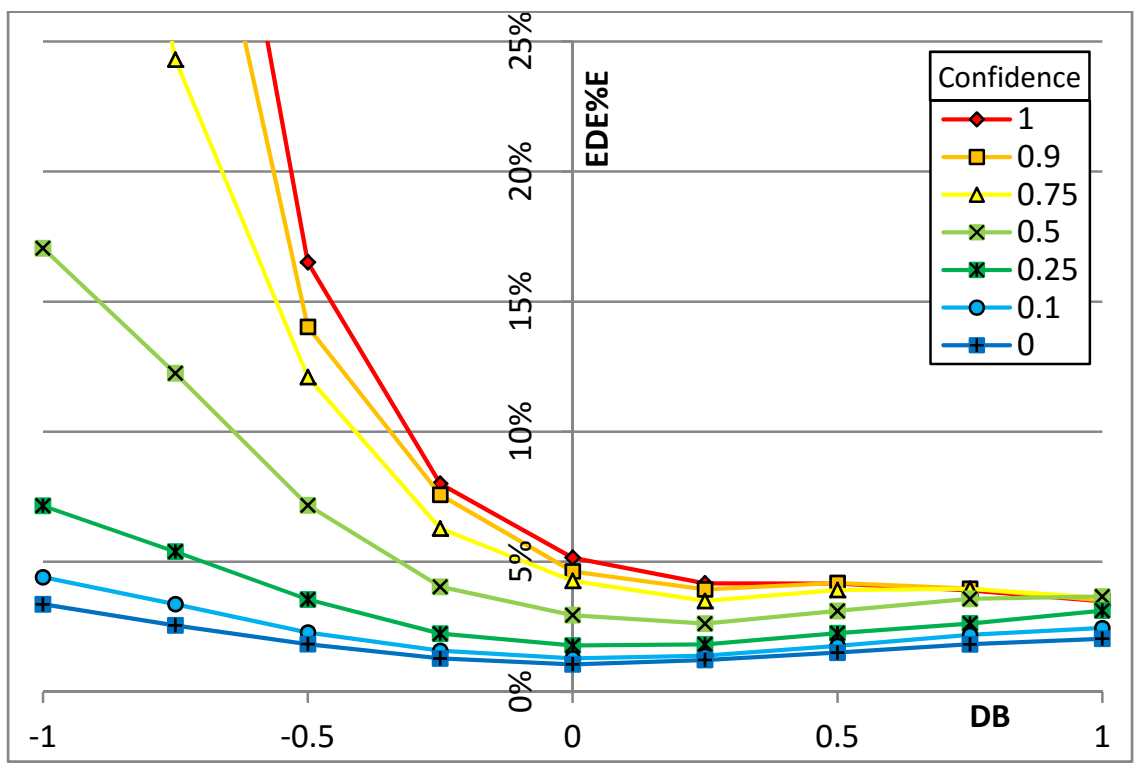

Fig. 14-Expected decision error, constrained budget with stochastic bias.

\section{Results Behavior}

$\mathrm{EDE} \% \mathrm{E}$ begins to decrease for cases with high overconfidence as directional bias approaches 1 in all EDE\%E plots (Figs. 8, 10, 12, and 14), but most pronouncedly in Fig. 8. This unusual behavior results because estimated EVs tend to infinity as bias approaches complete optimism and complete overconfidence (determinism). To study this phenomenon further, consider that the estimated NPV is in the denominator of $\mathrm{EDE} \% \mathrm{E}$, as in Eq. 17: $E D E \% E=E\left(\frac{B P N P V-R N P V}{E s t N P V}\right)$. The numerator has two terms: the best possible NPV, which is constant and is a property of the global portfolio characteristics, and the realized NPV, which will change with overconfidence and directional bias but will never exceed the BPNPV. The denominator, on the other hand, can increase exponentially with high values of overconfidence and directional bias as 
illustrated in Fig. 15, which focuses on the behavior of the EstNPV for high values of confidence and directional bias $(>0.5)$ in the unconstrained budget case. To break this down even further, the estimated NPV is $N P V=P V O C F-C a p E x$, and PVOCF keeps increasing as the values of overconfidence and directional bias approach 1, representing an increasing deterministic estimation on the long tail of a lognormal distribution for the PVOCF parameter. The CapEx parameter, at the same time, decreases significantly with high values of overconfidence and directional bias because it is a cost parameter. This allows the estimation process to squeeze in more projects, because they are estimated to have low CapEx, each with additional unrealistically high EstNPV.

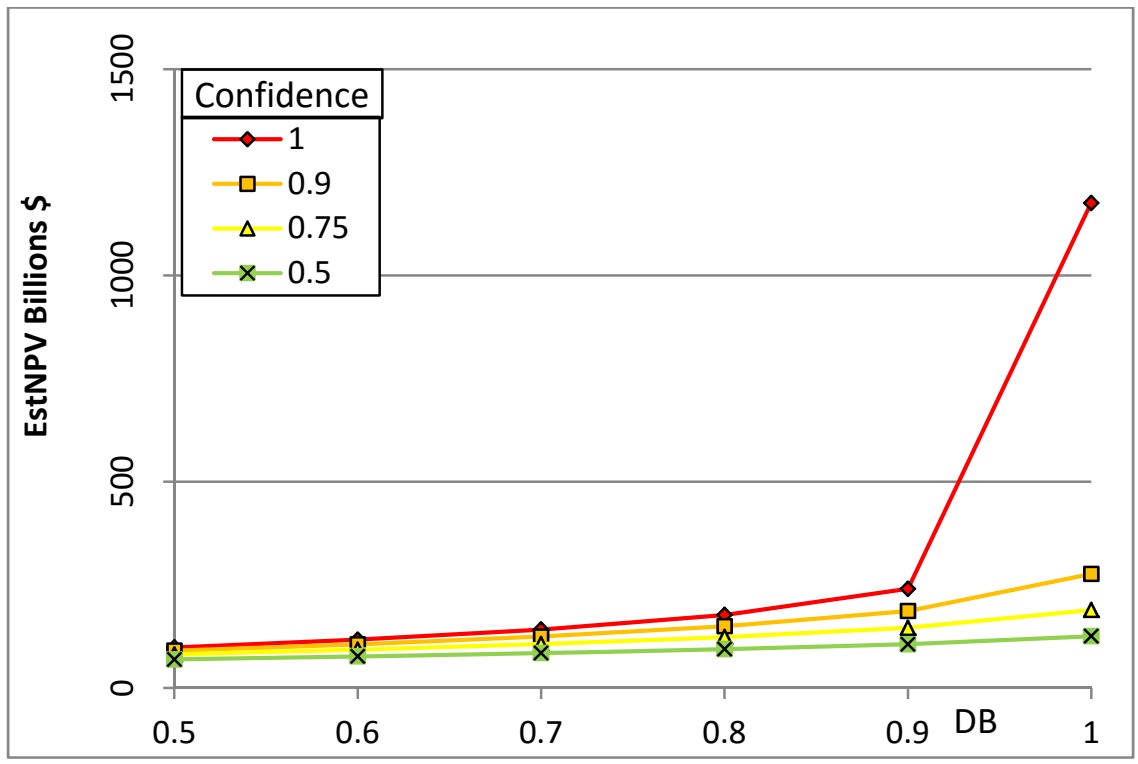

Fig. 15-Estimated NPV for high OC and DB. 
Fig. 16 shows the realized NPV for the same unconstrained case with high values of confidence and directional bias $(>0.5)$. The decreasing trend of the curves results from the effect of bias and overconfidence on the realized NPV of the portfolio. The flattening towards the very high end of the scale is due to the increasing occurrences of instances when the estimator will select all 100 projects of the simulated portfolio. Therefore, no additional penalty will occur in further increases in optimism or overconfidence.

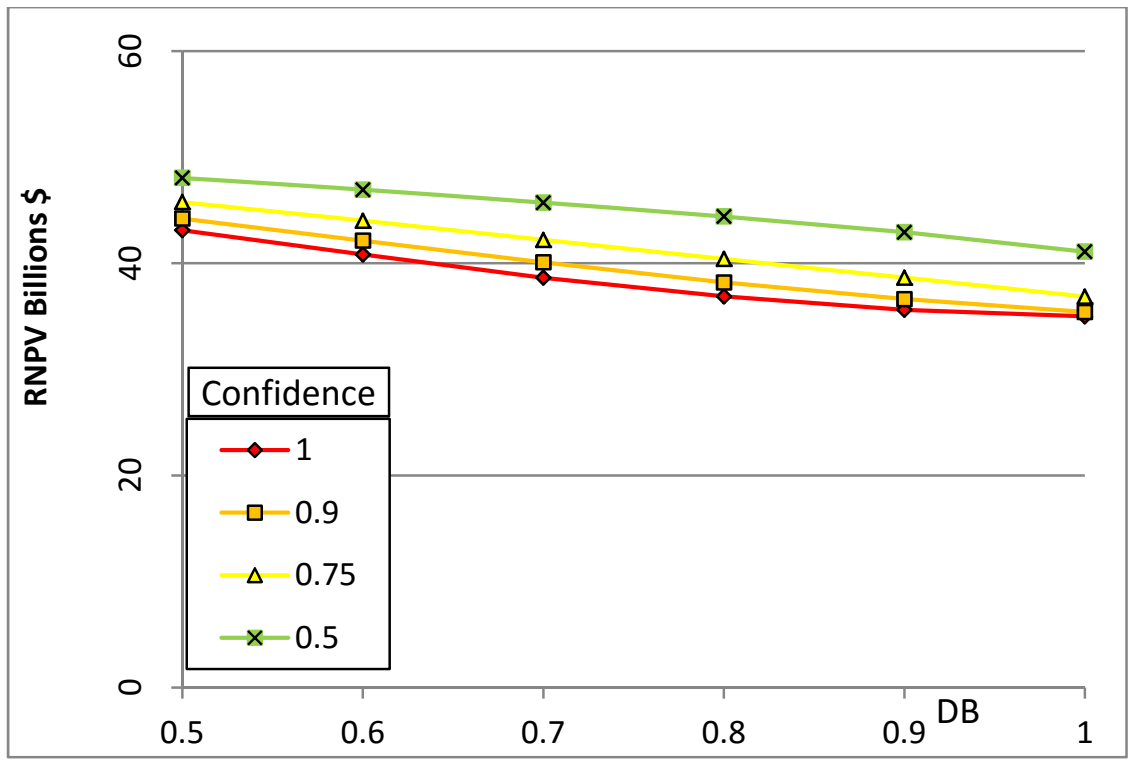

Fig. 16-Realized NPV for high OC and DB

\section{Discussion}

Results above indicate that, for companies that have traditionally conducted deterministic assessments, simply converting to probabilistic methods may not be enough. Going through the probabilistic motions and quantifying only part of the 
uncertainty may reduce the disappointment, but there will still be disappointment. For example, reducing overconfidence from 1 (deterministic) to 0.5 with optimistic directional bias of 0.5 for an unconstrained-budget selection will reduce $\mathrm{ED} \% \mathrm{E}$ from $56 \%$ to $31 \%$ (Fig. 7 ), but $31 \%$ of estimated NPV is still significant disappointment. What is needed to eliminate disappointment and decision error is reliable, or complete, assessment of uncertainty. When uncertainty is quantified reliably and consistently, the P90 will be realized $90 \%$ of the time, the P50 will be realized $50 \%$ of the time, and the P10 will be realized $10 \%$ of the time.

The model relating estimated distributions to true distributions is approximate and the modeling requires the input of a number of parameters - global distribution forms and parameters, true project distribution forms, numbers of projects, and budget limits. While I attempted to model portfolios typical of a large oil and gas company based on previous study, the parameters are largely assumed. The numerical results will vary if different inputs are assumed. Despite these limitations, the model is quite useful in quantifying the magnitude of potential impacts of biases on portfolio performance and the overall conclusion that this impact can be large is quite reasonable.

\section{Oil and Gas Estimation Process}

In this section, a typical oil and gas estimation process will be presented to link this framework back to oil and gas industry project evaluation. The applicability of the concepts presented can be extended to any field, but this section gives a practical example of how reservoir engineers might evaluate oil and gas fields probabilistically. 
After analyzing the reservoir input data and quantifying uncertainties, the reservoir engineers would produce CapEx and PVOCF curves similar to the ones studied in the previous chapter: "New Framework for Modeling Impact of Biases."

Company A has secured funding to invest in one or more prospects. One such prospect is a Coal Bed Methane field in its final pilot stage with six existing exploration wells producing from six months to one year (Fig. 17). Depths, pay thicknesses, gas contents, and permeability data, as well as analogous field data, are available to start scoping the project. The contract for this field requires a minimum investment of 50 drilled wells and the economics will be run for an expected life of 40 years.

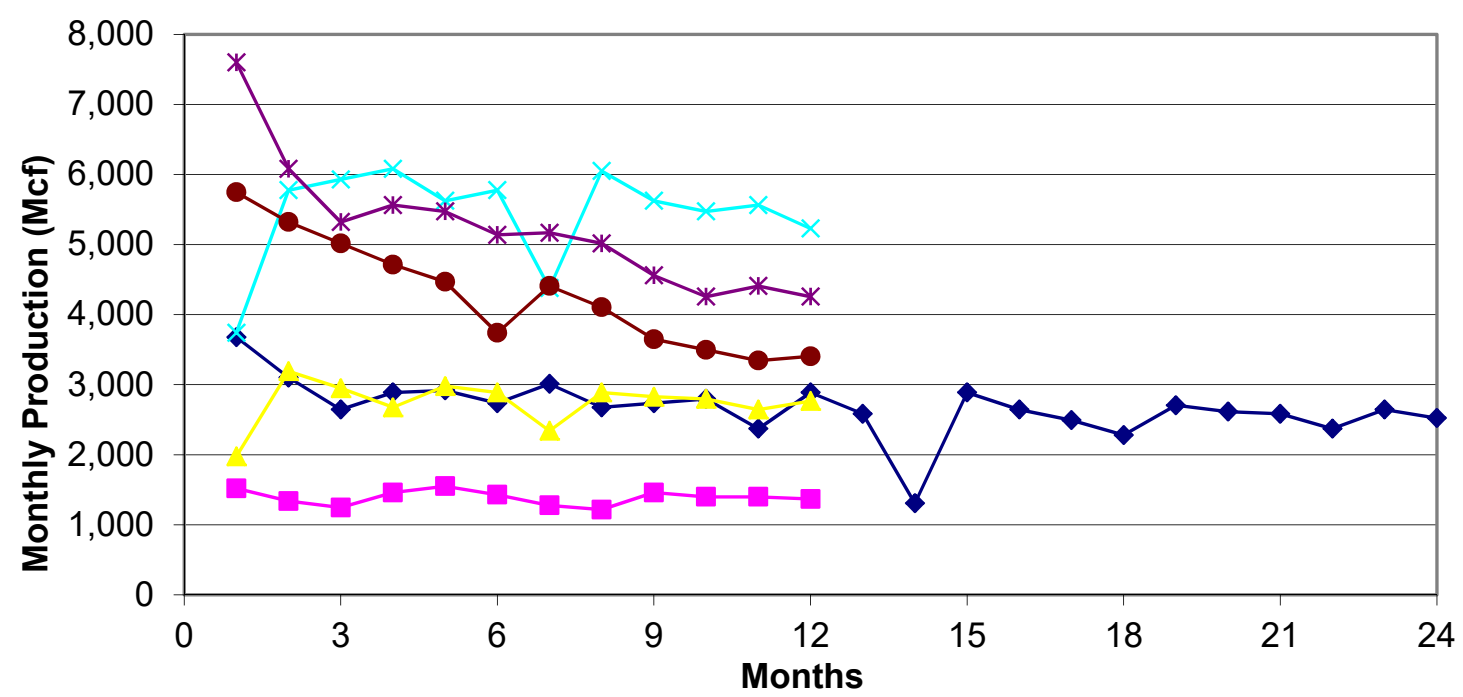

Fig. 17-Production profile of six pilot wells in Coal Bed Methane prospect 


\section{Data analysis}

Decline curve analysis was carried out on the six exploration wells to fit a general hyperbolic decline equation $q=q_{i}\left(1+b \times D_{i} \times t\right)^{\frac{-1}{b}}$ (Table 5).

\begin{tabular}{ccccc}
\multicolumn{5}{c}{ Table 5-Decline Curve Analysis of pilot wells } \\
Parameter & $\underline{\text { Mean }}$ & $\underline{\mathrm{P} 90}$ & $\underline{\mathrm{P} 50}$ & $\underline{\mathrm{P} 10}$ \\
\cline { 1 - 3 } & 183.83 & 70.70 & 158.05 & 327.27 \\
$q_{i}$ & 40.37 & 82.38 & 33.17 & 7.38 \\
$b$ & 17.85 & 2.54 & 9.27 & 39.56
\end{tabular}

Analogous fields show increased recovery efficiency with decreased wellspacing, i.e., $19 \%, 28 \%, 42 \%, 53 \%$, and $75 \%$ of original gas in place for well-spacings of $640,320,160,80$, and 40 acres/well, respectively. Plotting the field recovery factor (RF) per well (i.e., field recovery factor divided by well count) versus the number of wells needed to develop the 25,000 acres in this scenario shows that percentage of final field recovery contributed by each well is related to the square root of the number of wells (Fig. 18). In other words: $\frac{R F}{\text { wellCount }} \approx \frac{3.3 \%}{\sqrt{\text { WellCount }}}$, and this will allow calculating the recovery factor of the field for any given number of wells by simply multiplying a constant by the square root of that number of wells: $R F=3.3 \% \sqrt{\text { WellCount }}$. For example, 320 acre/well spacing requires 78 wells and gives $28 \%$ recovery factor. Fig. 18 shows a corresponding data point at 78 wells versus $0.36 \%$ field recovery factor per 
well, which came from $28 \%$ field recovery factor divided by 78 wells. The equation approximates the recovery factor by multiplying $3.3 \%$ by $\sqrt{78}$ and directly gives $29.1 \%$.

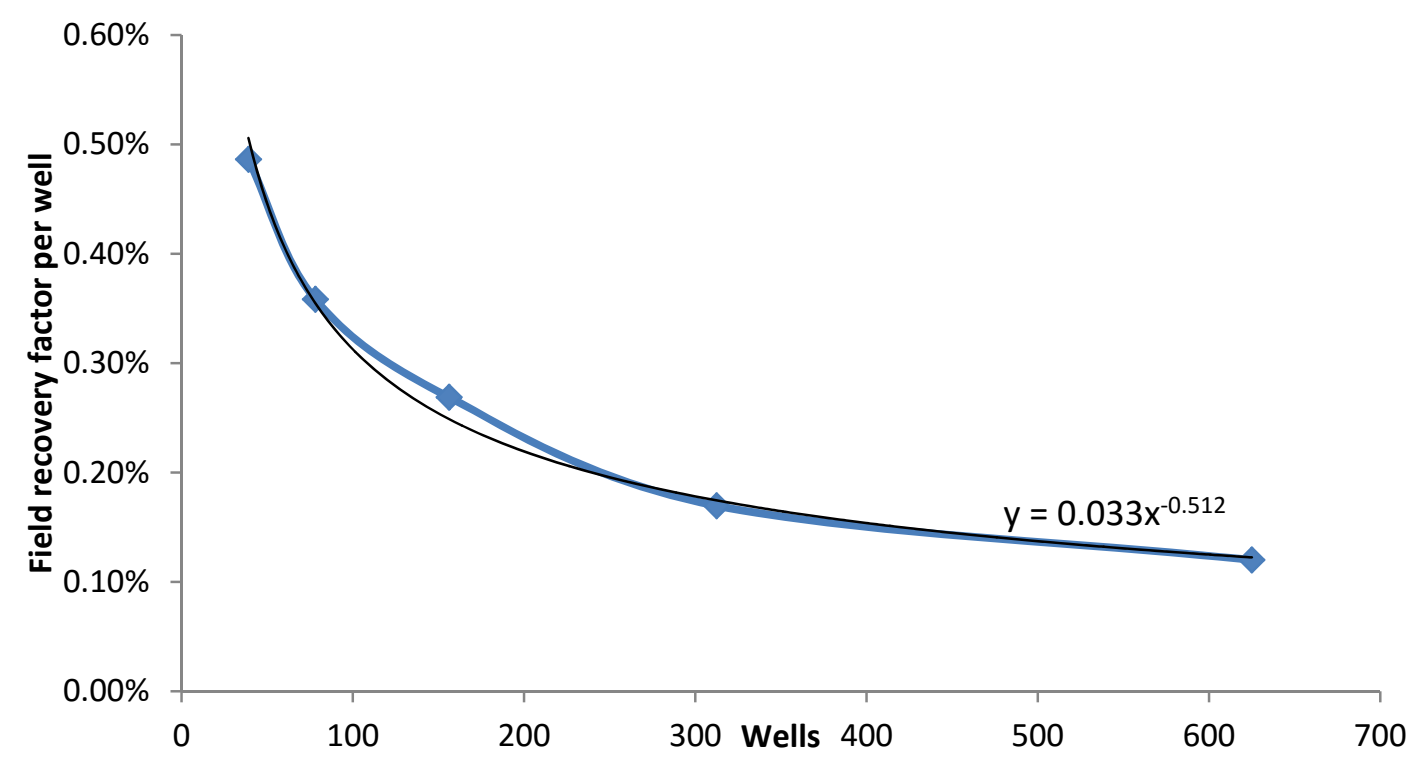

Fig. 18 - Field recovery factor per well decreases with smaller acre/well-spacing, i.e., increased number of wells

After analyzing the decline curves and finding an equation for the field recovery factor per well, it is possible to combine those two pieces of information to modify the DCA parameters to generate pseudo well rates that can readily be multiplied by the square root of the number of wells to give the total production rate of the field. Modification of the DCA parameters to achieve such pseudo well rates will not change the initial decline rate and the exponential component $\left(D_{i}\right.$ and $\left.b\right)$ parameters from the DCA study above (Table 5), but will only change the initial rate $\left(q_{i}\right.$ to $\left.q_{i}^{*}\right)$ to reflect the expected ultimate recovery. Modification of only the initial production rate assumes that increasing the number of wells will not change the shape of the production profile for 
each well but will change the overall quantity of production; i.e., it will shift the production curves on the $y$-axis but will not change their shapes. The final curves that will be generated using the DCA parameters are similar to the curves in Fig. 17 in shape, but the translation on the y-axis are designed to generate a total field production, EUR, over a 40-year field life that matches the EUR from reservoir data. The EUR from reservoir data is calculated by this equation: $E U R=R F * G I P$, where $\mathrm{RF}$ is calculated from Fig. $18(R F=3.3 \% \sqrt{\text { WellCount }})$ and GIP is calculated using this equation: GIP $=$ Area $*$ Density $*$ Pay $*$ GasContent $*(1-\phi)$. This equation utilizes reservoir data from other existing wells in the same field, namely the gas content (Fig. 19) and net pay (Fig. 20). Porosity is assumed to be a very low constant value of 0.03 and coal density is assumed to be $1.65 \mathrm{gm} / \mathrm{cc}$.

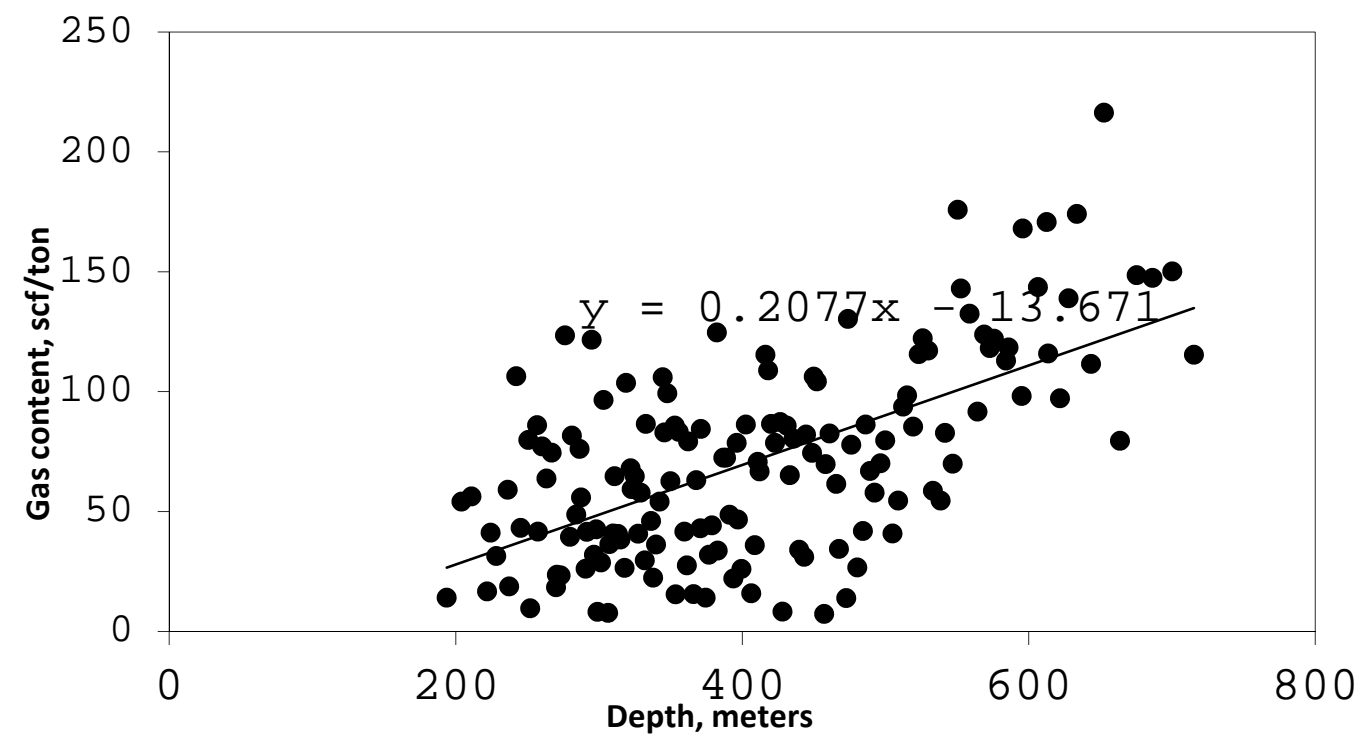

Fig. 19-Gas content versus depth from wells in the same field 


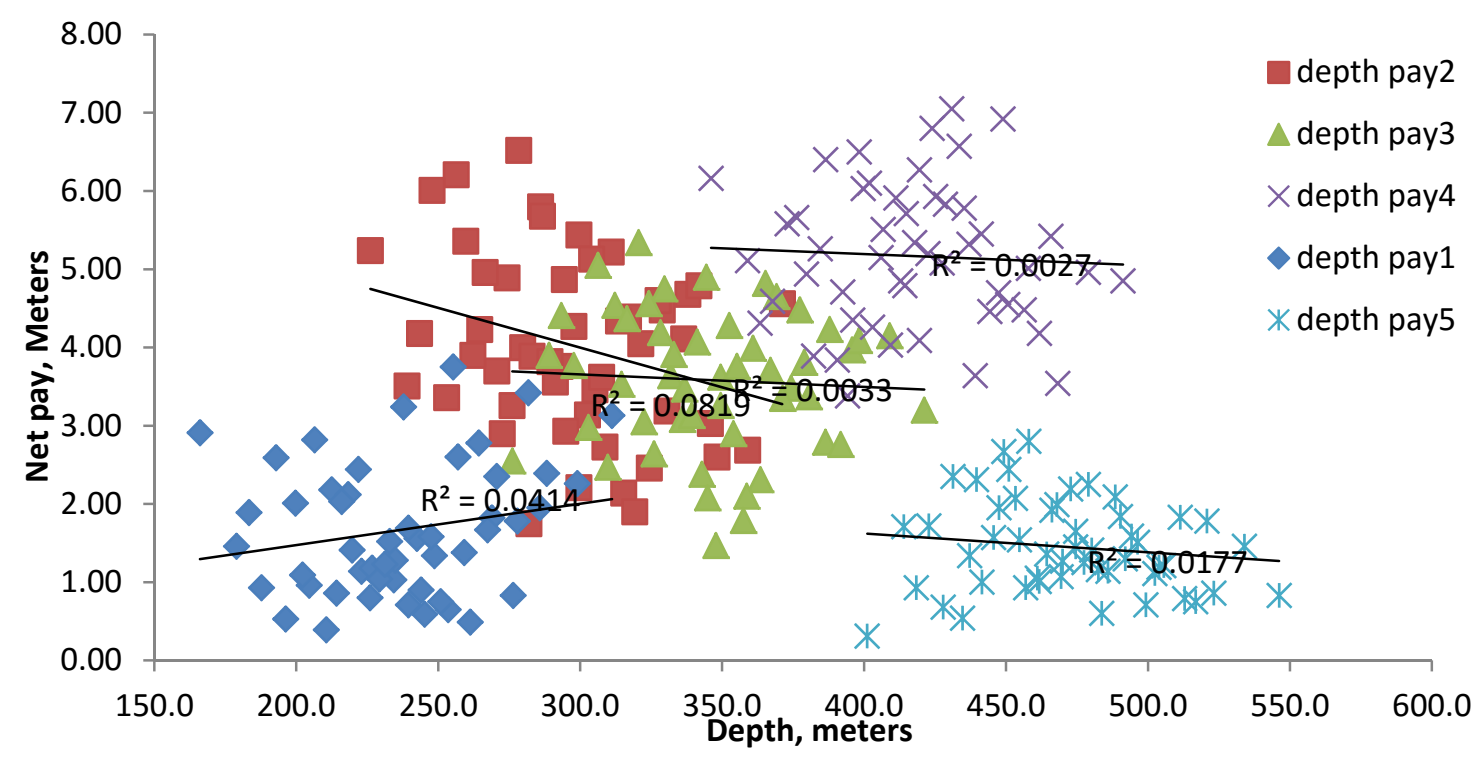

Fig. 20-Net pay versus depth for the five layers in the reservoir

To summarize the procedure so far, the production profile is estimated from the production history of existing wells, and an EUR is calculated at 40 years; let us call it

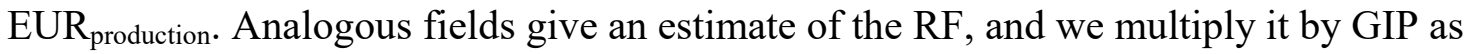
calculated from reservoir data from the field to get another estimate of the EUR; let us call it EUR reservoir. $_{\text {The production profiles are scaled to match EUR }}$ reservoir by changing the initial production rate from $q_{i}$ to $q_{i}^{*}$. The final result is a production profile, defined by $q_{i}^{*}, D_{i}$, and $b$, of all the wells in the field as a function of the number of wells. 
Surveyed cost for pipeline in this area shows decreasing cost per well for each well-spacing, i.e., $\$ 150 \mathrm{M}, \$ 120 \mathrm{M}, \$ 80 \mathrm{M}, \$ 60 \mathrm{M}$, and $\$ 40 \mathrm{M}$ for each well at spacings of $640,320,160,80$, and 40 acres/well, respectively. Plotting the pipeline cost per well shows a relationship between the square root of the number of wells and the cost (Fig. 21). The fitting equation is: $\frac{\operatorname{cost}}{\text { well }}=\frac{\$ 1 M M}{\sqrt{\text { well }}}$ or $\operatorname{cost}=\$ 1 M M \sqrt{\text { well }}$. For example, 160acre/well spacing requires 156 wells for this field, and costs $\$ 80 \mathrm{M} * 156=\$ 12.5 \mathrm{MM}$ as estimated from the same area. Surface pipeline costs are assumed to be independent of underground parameters and depend on the general cost in the vicinity of the development.

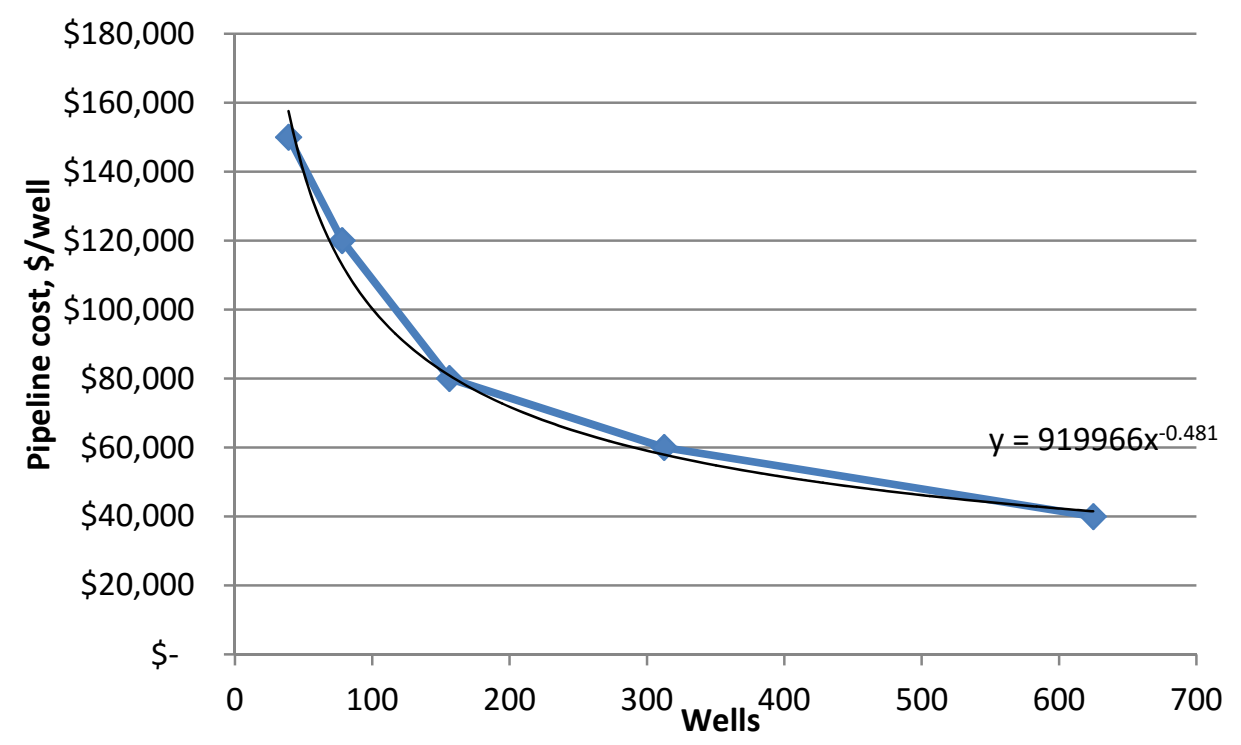

Fig. 21-Cost of pipeline tie-in from surveyed wells in the same area 


\section{Known unknowns}

Nine independent parameters were identified as the main sources of uncertainty in the estimation process (Table 6). Truncation was used for normal distributions to avoid negative results.

Table 6-List of sources of uncertainty in example estimation process

\begin{tabular}{|l|l|l|}
\hline & Sources of Uncertainty & Distribution fit \\
\hline 1 & Pseudo initial daily gas rate $\left(q_{i}^{*}\right)$ Mscf/Day & Lognormal(Mean 1500, SD 500) \\
\hline 2 & Yearly decline rate $\left(D_{i}\right), \% /$ year & Lognormal(Mean 150, SD 150) \\
\hline 3 & Exponential base $(b)$, dimensionless & Lognormal(Mean 20, SD 5) \\
\hline 4 & Drilling cost per well, \$ & Normal(Mean \$200M, SD \$20M)>0 \\
\hline 5 & $\begin{array}{l}\text { Facilities cost in \$ per 5000 Mscf/Day } \\
\text { increments }\end{array}$ & Normal(Mean \$5MM, SD \$1MM)>0 \\
\hline 6 & Adjusted gas price after royalties in \$/Mscf & Normal(Mean 5, SD 1)>0 \\
\hline 7 & Fixed OpEx in \$ per well per year & Normal(Mean \$10M, SD \$1M)>0 \\
\hline 8 & Variable OpEx in \$ per Mscf & Normal(Mean \$0.15, SD \$0.05)>0 \\
\hline 9 & Abandonment cost in \$ per well & Normal(Mean \$50M, SD \$10M)>0 \\
\hline
\end{tabular}

To keep this model simple, other sources of uncertainty were defined in terms of one or more of the nine factors in Table 6. For example, volumetrics and ultimate recovery were accounted for by the initial well rate $\left(q_{i}^{*}\right)$ in the pseudo well rate. The equations for finding the RF and the cost of pipelines are only valid for more than 50 
wells (Fig. 18 and Fig. 21), and 50 wells is the minimum number of wells required to develop this field without losing the license.

\section{Estimation process}

The project life was estimated to be 40 years, after which the wells will be abandoned. All capital expenditures are assumed to happen in year 0 , including drilling of all the wells, pipelines and facilities. No production is assumed for year 0 , and the initial well rate posted in the calculations phase for year 0 represents the initial well rates at the end of the year and is only used to calculate the production during the next year, year 1 . During the lifetime of the project, the well rates are calculated and accumulated per year, and from that, the costs and income are calculated on a year-by-year basis. Operating cash flows are discounted at 15\%. Monte-Carlo simulation was carried out on the parameters in Table 6 to estimate the costs and income. Sample numbers from one Monte Carlo iteration for capital expenditure are shown in Table 7, for field production in Table 8, and for operating cash flows calculations in Table 9. Table 7 lists the cost of drilling the wells, the cost of the pipelines and the tie-ins, and the cost of the facilities as predicted by this one Monte-Carlo iteration. The total of those three costs is the CapEx and they are not discounted because they are all incurred in year 0 . Table 8 lists the pseudo well rate at the end of that year, the yearly production of a single pseudo well, and the total field production. The daily production rate of the total field is not listed, but can be obtained by multiplying the single pseudo well rate by the square root of the wells. The cumulative production of a pseudo well is listed from the beginning of year 1 until the end of the specific year number. The field cumulative production is also not 
listed but can be obtained by multiplying pseudo well production by the square root of the number of wells. This cumulative field production will keep increasing until it reaches EUR at end of year 39. The following column is the actual production volume for that specific year, and it is calculated by taking the difference between the two cumulative pseudo productions of the current and past year and then multiplying the difference by the square root of the number of wells. The revenue in Table 9 is simply the gas price times the yearly field production. OpEx are calculated separately for fixed and variable expenditures. The Operating Cash Flow (OCF) is the revenue minus the two OpEx's.

Table 7-Capital expenditures (samples from one Monte Carlo iteration)

$\frac{\text { Wells }}{\$ 26,144,199} \quad \frac{\text { Pipelines }}{\$ 12,529,964} \quad \frac{\underline{\text { Facilities }}}{\$ 10,872,247} \quad \frac{\underline{\text { CapEx }}}{\$ 49,546,410}$

Table 8-Field production calculations (samples from one Monte Carlo iteration) Year Number $\quad$ vol $/$ day $/ \sqrt{\text { well }(M s c f d)} \quad \underline{\text { Cum } / \sqrt{\text { well }} \text { (Mscf) }}$ Vol/year (Mscf)

$\begin{array}{llll}0 & 1265.3 & 0.0 & 0.0 \\ 1 & 710.0 & 280664.9 & 3516721.2 \\ 2 & 673.5 & 532502.5 & 3155515.4 \\ 3 & 652.9 & 774462.8 & 3031754.9 \\ \ldots & \ldots & \ldots & \ldots \\ 38 & 537.9 & 8082542.8 & 2464179.7 \\ 39 & 536.8 & 8278811.0 & 2459233.5 \\ 40 & 0.0 & 0.0 & 0.0\end{array}$




\section{Table 9-Operating cash flow calculations (sample from one Monte Carlo iteration)}

\begin{tabular}{|c|c|c|c|c|}
\hline Year Number & $\underline{\text { Revenue }}$ & Fixed & Variable & $\underline{O C F}$ \\
\hline 0 & $\$$ & $\$$ & $\$$ & $\$$ \\
\hline 1 & $\$ 19,858,064$ & $\$ 1,678,816$ & $\$ 350,847$ & $\$ 17,828,401$ \\
\hline 2 & $\$ 17,818,424$ & $\$ 1,678,816$ & $\$ 314,811$ & $\$ 15,824,797$ \\
\hline 3 & $\$ 17,119,578$ & $\$ 1,678,816$ & $\$ 302,464$ & $\$ 15,138,298$ \\
\hline$\ldots$ & $\ldots$ & $\ldots$ & $\ldots$ & $\ldots$ \\
\hline 38 & $\$ 13,914,620$ & $\$ 1,678,816$ & $\$ 245,840$ & $\$ 11,989,964$ \\
\hline 39 & $\$ 13,886,690$ & $\$ 1,678,816$ & $\$ 245,346$ & $\$ 11,962,528$ \\
\hline 40 & $\$$ & $\$$ & $\$$ & $\$(6,444,486)$ \\
\hline
\end{tabular}

Simulation results for valid well spacings are shown in Fig. 22. Both PVOCF and CapEx are skewed to the right. The portfolio modeling experiments presented earlier assume that Company A would conduct similar evaluations for 100 different investment prospects available in the market. For each of those 100 estimated projects, Company A will have distributions of CapEx and PVOCF similar to those in Fig. 22, and then they can choose the optimal portfolio within their capital budget. 


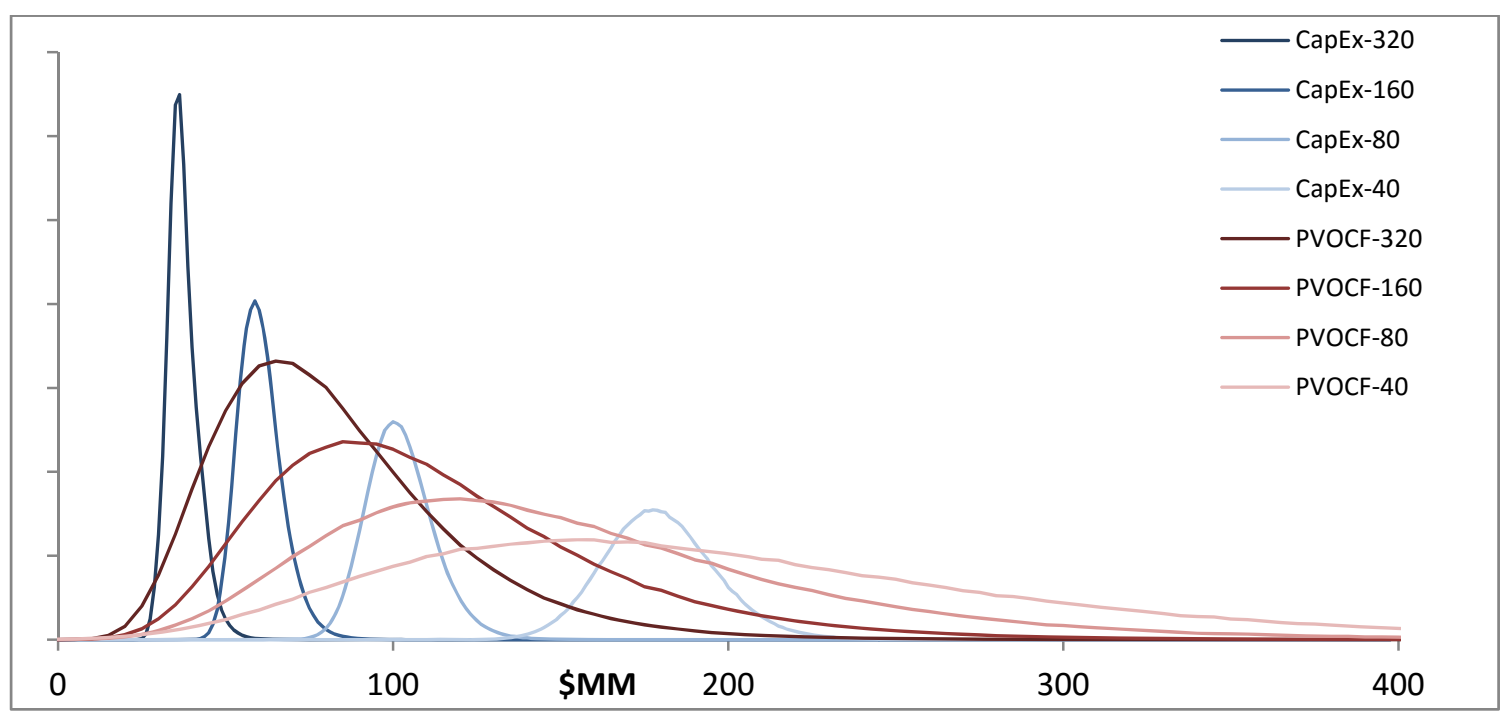

Fig. 22 - Probability Density Function result of four typical project evaluations based on well spacing (acres/well)

\section{Sources of overconfidence and directional bias}

Overconfidence and directional bias can affect the estimation process from two sources.

Parameters that have not been captured, unknown unknowns, are the uncertain

parameters that were not taken into consideration in the estimation process. The second source of overconfidence and directional bias are the parameters that have been captured, but not fully or with a bias.

Examples of unknown unknowns that were not considered in this study and can increase the CapEx estimation are regulations overhead, delays in the infrastructure construction or drilling, and limited availability of drilling rigs, equipment or skilled crew. 
Examples of parameters that were considered but may not have captured the true underlying uncertainty correctly are: the probability that the drainage area is larger/smaller than originally assumed, the possibilities of super productive wells, and huge fluctuations in the oil or gas price (for example, fluctuations that occurred in 1998 and 2008). Estimators with high (or low) directional bias will possibly not capture the mean or the central tendency of those parameters correctly, estimators with high overconfidence will possibly not capture the dispersion of such parameters correctly, and estimators with a combination of overconfidence and directional bias will possibly not capture both correctly. 


\section{DISCUSSION}

\section{Is Inevitable Disappointment Inevitable?}

Various authors have reported the phenomenon termed the Optimizer's Curse, Inevitable Disappointment or Post-Decision Surprise (Begg and Bratvold, 2008; Smith and Winkler, 2006). This is claimed to be a systematic bias resulting from the decision process itself. Because the decision process involves the selection of more highly valued alternatives from a set, random errors in the estimation of project values result in projects estimated on the high side to be selected with greater frequency than projects estimated on the low side. This results in a positive bias in estimated value, which results in disappointment when the actual lower value is realized. Authors claim the positive bias is present even when the project estimates are unbiased.

Results presented earlier have shown that Inevitable Disappointment is not really inevitable. First of all, disappointment can be negative, on average, which means the operator will experience pleasant surprise rather than disappointment when the actual values are realized. This can occur when there is pessimism combined with overconfidence, as shown in the simulation results above. While this situation may not be common in practice, it is possible. Negative expected disappointment was also observed by Begg and Bratvold (2008).

\footnotetext{
* Reproduced with permission of Society of Petroleum Engineers (SPE) to reuse SPE-160189 "The Value of Assessing Uncertainty," doi:10.2118/160189-PA. Copyright 2014, Society of Petroleum Engineers Inc. Further reproduction prohibited without permission.
} 
Results also refute two claims in the literature, one that Inevitable Disappointment is inherent in the process itself, and another that it happens even when there is no bias. Inevitable Disappointment results from random estimation errors, but the source of this randomness is estimator biases, particularly overconfidence, which are very often present in limited-resources assessments. This model shows that with no estimator biases (zero overconfidence and zero directional bias), expected disappointment is zero (Fig. 7 and Fig. 9) because estimated EVs equal true EVs. Estimator biases are required to achieve non-zero expected disappointment. For example, adding overconfidence but no directional bias results in non-zero $\mathrm{ED} \% \mathrm{E}$ (because equal truncation from both ends of lognormal distributions does not preserve the means), but in the studied cases it is not disappointment (positive ED\%E) but rather pleasant surprise (negative $\mathrm{ED} \% \mathrm{E}$ ). It takes optimism in combination with overconfidence to produce even small amounts of positive expected disappointment. It should be pointed out that this result may be tied to the particular portfolio parameters used in this work and may not be true in general. That is, it may be possible to produce positive expected disappointment with overconfidence and zero direction bias with different portfolio parameters.

If Inevitable Disappointment results from estimator biases, this means that it can be driven to zero with the elimination of the estimator biases. This is shown in the results (Fig. 7 and Fig. 9); ED\%E goes to zero as overconfidence is eliminated (confidence goes to 0 ) regardless of the directional bias. While it is unlikely that random estimation errors can be completely eliminated in practice, these results do suggest that expected 
disappointment, as well as expected decision error, can be driven to negligible levels with good uncertainty quantification practices, i.e., practices that result in estimated project value distributions that are close to the true distributions.

\section{Relationship of Biases Impact to Industry Performance}

It is of interest to examine the relationship between expected portfolio disappointment and financial performance. This can be done using simple cash-flow analysis for the constrained-budget case with an investment of \$5B. For simplicity, let us assume estimated equal annual cash flows from the portfolio of $\$ 996 \mathrm{MM}$ over a 10 -year period. This yields an internal rate of return of $15.0 \%$ and an NPV of $\$ 1120 \mathrm{MM}$ discounted at $10 \%$, reasonable goals for the portfolio on average. With moderate amounts of overconfidence and optimism ( 0.5 and 0.5$), \mathrm{ED} \% \mathrm{E}$ is $31 \%$ of estimated NPV for the constrained-budget case (Fig. 9). This corresponds to reducing the true NPV to \$773MM, which can be achieved by reducing the actual annual cash flows from \$996MM to \$940MM, yielding an IRR of $13.5 \%$.

As stated earlier, Brashear et al. (2001) reported that in the 1990s the return on net assets by the largest U.S.-based E\&P companies was only 7\%, after selecting projects with hurdle rates of generally $15 \%$ or more, which were all financed with cost of capital generally in the range of 9-12\%. Using our simple cash-flow analysis, we can achieve a 7\% IRR by reducing the actual annual cash flows to $\$ 713 \mathrm{MM}$, which results in a true NPV of $-\$ 619 \mathrm{MM}$ and an ED\%E of $155 \%$. This implies extremely high values of overconfidence and optimism. However, ED\%E values this high were not reached in the modeling; the highest $\mathrm{ED} \% \mathrm{E}$ experienced in any of the cases was around $100 \%$ (Fig. 7 
and Fig. 9). Earlier in this work, it was shown that expected disappointment depends on the properties of the true global portfolio, and that a more negative global portfolio (greater fraction of projects with true mean NPV less than zero) yielded greater disappointment (Table 1). Both Begg and Bratvold (2008) and this study chose global portfolio properties that were quite positive; i.e., only a relatively small fraction of the projects, $26-28 \%$, had true distributions with significant probability of loss. With these global portfolio properties and 100 projects to choose from, it is virtually impossible to experience an overall portfolio loss. $\mathrm{ED} \% \mathrm{E}$ values of $150 \%$ or more can be achieved by making the true global portfolio significantly more negative (majority of projects with true NPV $<0$ ) and using high values of overconfidence and optimism. Since the industry apparently experienced an overall loss in the 1990's, it appears that both Begg and Bratvold and I were guilty of both overconfidence and optimism in our choices of global portfolio properties in modeling the impact of biases.

Little recent commentary was found in the literature about poor industry financial performance. While one might conclude that this is the result of significantly better uncertainty quantification practices, the evidence suggests otherwise, e.g., Merrow (2011). Continued overconfidence and optimism were likely masked by high oil prices in the last decade. While the industry may have performed better than the previous decade, one can suspect that it did not perform as well as predicted.

If significant overconfidence and optimism resulted in a $7 \%$ return in the $1990 \mathrm{~s}$ when predicted returns were $15 \%$ or greater, does this imply that elimination of these biases would have increased industry performance from $7 \%$ to $15 \%+$ ? Elimination of 
overconfidence and optimism would eliminate decision error and disappointment, but it would not necessarily increase industry performance, although it is possible. It is also possible that eliminating overconfidence and optimism may simply lower the potential returns of all project choices. Thus, we would still experience lower returns, but we would not be surprised by it. Of course, eliminating overconfidence and optimism, and thus disappointment, would help us avoid the situation described in Brashear et al. (2001); we would not consistently invest in projects that return less than the cost of capital. Overall industry financial performance will increase only if there are some projects that have significantly greater true potential performance and better quantification of uncertainty allows us to identify and select these superior projects.

\section{What to Do?}

The case has been made that overconfidence and optimism are chronic, that they go hand in hand, and that combined they have a significant negative impact on the performance of individual company portfolios and the industry overall. The next question is what should be done about it.

As stated earlier, overconfidence is the most significant of the biases and is the first cause of poor estimation and project evaluation. It is only with overconfidence, the failure to consider all the possible outcomes of a project, that other biases are able to flourish and impact estimation. For example, anchoring to a high initial NPV estimate can lead to an optimistic estimated EV only if overconfidence prevents consideration of possible negative outcomes that counter the initial high estimate. If overconfidence is the 
first cause, then overconfidence should be the primary focus of mitigation efforts. Elimination of overconfidence involves considering all the possible outcomes of a project. Considering all the possible outcomes of a project automatically corrects for other biases that alter the central tendency of the estimate (directional bias). This is demonstrated in the results shown earlier; reducing overconfidence (reducing the confidence parameter) with a fixed value of the directional bias parameter reduces ED\%E and EDE\%E (e.g., Fig. 7 and 8). This is consistent with Capen (1976) assessment that a better range leads to a better mean.

It is relatively easy to consider the outcomes one can think of. It is difficult to consider the outcomes that do not even come to mind, the unknown unknowns. The key to eliminating overconfidence is accounting for these unknown unknowns. The best way to do this is through calibration, a process of tracking probabilistic predictions and comparing how actual performance turns out relative to the predictions (look-backs). This is not difficult. However, it requires discipline, corporate memory, and an appreciation for the value of uncertainty quantification, which is why it is not commonly practiced.

Results have demonstrated that the cost of underestimating uncertainty (disappointment) can be quite large, which implies that the value of quantifying uncertainty reliably can also be quite large. However, the cost of estimating uncertainty reliably has not been addressed, which must be less than the value to be worthwhile. It is not necessary to adopt expensive, probabilistic decision-analysis practices throughout an organization. What is required is a commitment to reliable uncertainty quantification, a 
systematic look-back process, and appropriate levels of probabilistic decision-analysis being adopted where it is needed - simple methods for some assets, more complex systems for larger, higher-cost assets.

\section{Measuring Confidence and Directional Bias}

Measuring the confidence parameter of any estimator can be achieved by following the calibration methodology of Capen (1976). His results were used to directly measure the confidence parameter in the community of typical petroleum engineers when this parameter was first introduced earlier in this work (Section "Intuitive description," page 18). Enhancement to the survey questions or methodology has been suggested to tailor the measurements to the oil and gas industry or a specific company (Hawkins et al., 2002; Welsh et al., 2005).

Measurement of the directional bias is not as straightforward. Optimism has been studied in the psychology field a lot more than in the oil industry. For example, while OnePetro lists only 500 publication with the term "optimism," psycINFO database of the American Psychological Association lists more than 7000. Two good reviews of hundreds of those papers can be found in two classic books: Matlin and Stang (1978) and Taylor (1989). While studying such references, terminology can be a major challenge because overconfidence is sometimes used interchangeably with optimism, while over-precision, defined as a type of optimism, is used to mean overconfidence (Moore and Healy, 2008). In addition, categorization can also be a challenge because many authors subcategorize optimism independently and differently. For example the following list was encountered (in alphabetical order): big and little optimism (Peterson, 
2000), comparative optimism (Radcliffe and Klein, 2002), dispositional optimism (Scheier and Carver, 1987), optimism bias (Flyvbjerg, 2006), optimism vs. neuroticism (Scheier et al., 1994), realistic optimism (Schneider, 2001), strategic optimism (Spencer and Norem, 1996), and unrealistic optimism (Weinstein, 1980). In this section, I focus on the measurement of optimism, and only three technical definitions of optimism were found that had published approaches to measurement in the psychology field, and they will be reviewed next.

The first technical definition of optimism is the simple desire of favorable outcomes and, at the same time, disinclination of unfavorable ones. Based on this definition, Life Orientation Test (LOT) was devised by Scheier and Carver (1985). The test has a set of positive questions, such as "In uncertain times, I usually expect the best," some negative questions, such as "If something can go wrong for me, it will," and some filler questions, such as "I enjoy my friends a lot." Each question is evaluated from strongly agree, 1 , to strongly disagree, 5 , and then the score of the positive and negative questions are used for a measure of optimism. This test has been enhanced in several subsequent publications, for example Life Orientation Test - Revised (LOT-R) (Scheier et al., 1994), and Extended Life Orientation Test (ELOT) (Chang et al., 1997).

The second technical definition of optimism is feeling in control of future events based on past experience, and pessimism as the feeling of helplessness based on past experience. Attributional Style Questionnaire (ASQ) defines an optimist as someone who has the ability to rationalize the causes of desirable outcomes to be internal, stable, and global, while the causes of undesirable outcomes to be external, unstable, and 
specific (Peterson et al., 1982). To measure this kind of optimism, fictional events (divided between good or bad) are described to participants, for example "you get a raise," and then they are asked about the expected cause (attribution) of each event, which in turn will be evaluated on the three dimensions: internality, stability, or globality (Peterson, 1991). This test was extended in CAVE (Content Analysis of Verbatim Explanations) to evaluate existing content media (text or audio) by extracting key information and presenting the extracts to judges who in turn evaluate the contents on the same three dimensions (Peterson and Seligman, 1984; Smith, 1992).

The third technical definition of optimism is a two-part definition of hope. One part is the ability to initiate actions and pursue goals (Agency), and the second part is the perception that there exists a way to achieve any goal (Pathways) (Snyder et al., 1996). I interpret this definition as a reflection of the proverb: "where there is a will, there is a way," where agency is having the will and pathways is finding the way. The test asks questions to participants in each of the two parts, for example, "I energetically pursue my goals," (Agency), and "There are lots of ways around any problem" (Pathways). The response to each question is then rated from 1 to 8 and summed independently for the two categories to create sub-scores and together to create the combined hope score (Peterson, 2000).

Unfortunately, most of the measurement studies focused more on establishing the metrics and correlating the optimism scores with health and well-being rather than getting a general optimism score for different types of professionals such as engineers, managers, or experts. The best prospect for getting a simple number for optimism (like 
0.644 for overconfidence) is Atalaya (2012), who casually published detailed LOT results, along with the internal consistency data and ANOVA calculations like others. The mean LOT score for 80 members of the Faculty of Economics and Administrative Sciences of Dumlupinar University was 3.84. The LOT scores are measured on a scale from 1 to 5 , where 1 is complete pessimism and 5 is complete optimism. Therefore, 3.84 translates to a directional bias score of 0.42 on a scale from - 1 to 1 using linear interpolation: $\frac{y-y_{0}}{x-x_{0}}=\frac{y_{1}-y_{0}}{x_{1}-x_{0}} \Rightarrow \frac{3.84-1}{x-(-1)}=\frac{5-1}{1-(-1)} \Rightarrow x+1=2.84 * \frac{2}{4} \Rightarrow x=0.42$. This is a generally optimistic faculty and if we assume a general confidence level of 0.644 then should expect 35\%-40\% disappointment by linear interpolation of Fig. 13, and 3\%$4 \%$ decision error by linear interpolation of Fig. 14. Before utilizing this result, two factors should be studied to assess its validity: first, the difference between the three reviewed definitions of optimism and the directional bias, and second, the implication of positive versus negative optimism score. The remainder of this section will address those two questions, and further discussion will be addressed in the Future Work section.

Regarding the differences between the three definitions of optimism and the definition of directional bias, the closest optimism definition to the directional bias parameter as used in this work is the one proposed by Flyvbjerg (2006), which is: "a cognitive predisposition found with most people to judge future events in a more positive light than is warranted by actual experience." This definition is closer to the LOT than ASQ or hope because it depends less on past experience. Both ASQ and the pathways part of hope depend more on past experience while Flyvbjerg's definition has more to do with current and future outlook, just like LOT's definition. What this means 
is that even though the LOT test does not measure directional bias directly, it is a better candidate to represent a measure of directional bias than the other two.

The second important factor to consider is the implication of reporting a highly optimistic score, or positive directional bias. Results of this work suggest that highly optimistic directional bias score leads to higher disappointment and decision error. On the contrast, the three reviewed optimism tests correlate high optimism scores positively with success in life, as demonstrated by original authors of those tests and others (Peterson, 2000). For example, high (LOT) optimism scores correlate positively with health, happiness and active coping (Scheier et al., 1986). High (ASQ) optimism scores correlate positively with athletic and academic success, and good health (Peterson and Park, 1998). Lastly, high hope-optimism scores correlate positively with high selfesteem, achievements, coping and positive emotions (Curry et al., 1997). Nonetheless, other authors have focused on the negative correlation between well-being and optimism, although still defined differently than directional bias. The classical example in this field is Henryism, which is based on a man with humble means who worked hard to provide for his children at the expense of his own health. His story transcended into a story about a skilled man who wanted to prove he can work faster than the steam machines that can replace him. The story ends with him winning over the machine because of his strong will, high skills, and optimism but immediately dying afterwards. This school recognizes that high levels of unfounded optimism and ambition lead to shorter life expectancy (James et al., 1987). Another example is when Oettingen (1996) 
described a type of unrealistic optimism that correlates negatively with quality of life in different aspects, such as health and love. 


\section{FUTURE WORK}

Underconfidence can be defined as the opposite of overconfidence. Extreme overconfidence leads to considering smaller and smaller parts of the information available, down to deterministic estimation. Underconfidence, on the other hand, will consider distributions wider than the true distribution, and extreme underconfidence will lead to a uniform distribution of all possible outcomes. A different view of the underconfidence parameter is increasing the deviation or dispersion of a random parameter to beyond its possible values that were based on previous information. This work considered only overconfidence. Underconfidence, although not as common, should also be investigated.

Industry performance in the 1990's was not matched using global portfolio distributions information available in the literature. A different global portfolio with better references should be used with the same methodology to match the 1990's performance. One good example is "U.S. Oil \& Gas Company Performance Report, Third Edition, Pennwell, Tulsa, Oklahoma, 1999," as referenced in Brashear et al. (2000).

A preliminary study of different definitions of optimism, along with questionnaires that measure those specific types of optimism, was presented in the section "Measuring Confidence and Directional Bias," page 76. To expand on this field, a survey should be created that measures directional bias directly. The test should have two parts, one for measuring indicative values of directional bias in the industry, and a 
second part that asks about future events that can be verified or inferred. For the first part, questions from one or more of the three tests presented can be used, and then the scores should be converted to the directional bias scale as demonstrated in that same section. For the second part about future events that can be verified, Puri and Robinson (2007) can be used as a reference because they created a test that asks about life expectancy, which can be inferred from actuarial tables. 


\section{SUMMARY*}

Evidence suggests that overconfidence and optimism in petroleum project evaluation are chronic and that they are usually present together. An approximate mathematical model to assess the impact of these biases on portfolio performance has been introduced, and some assumptions regarding the model input data for representative industry portfolios were made. Despite these limitations, the model is quite useful in assessing the impact of underestimation of uncertainty on portfolio and industry performance.

Modeling the impact of overconfidence and optimism demonstrates that, in combination, they can have significant impact on the performance of individual company portfolios. Typical overconfidence $(50 \%)$ and moderate amounts of optimism $(50 \%)$ resulted in expected disappointment of $30-35 \%$ of estimated NPV for the industry portfolios and optimization cases analyzed. Expected decision errors were small, 1-5\% of estimated NPV, for the same portfolios and cases. Greater degrees of overconfidence and optimism resulted in expected disappointment approaching 100\% of estimated NPV. Comparison of modeling results with industry performance in the 1990s suggests that these greater degrees of overconfidence and optimism were indeed experienced in the industry.

\footnotetext{
* Reproduced with permission of Society of Petroleum Engineers (SPE) to reuse SPE-160189 "The Value of Assessing Uncertainty," doi:10.2118/160189-PA. Copyright 2014, Society of Petroleum Engineers Inc. Further reproduction prohibited without permission.
} 
Reducing overconfidence reduces expected disappointment even if directional bias (optimism) remains fixed. Thus, expected disappointment can be reduced by focusing primarily on elimination of overconfidence; other biases are mitigated automatically in the process. The value of reliably quantifying uncertainty is the elimination of expected disappointment, which can be a substantial percentage of estimated NPV, and expected decision error. Elimination of expected disappointment will improve industry performance overall to the extent that superior projects are available and better quantification of uncertainty allows identification of these superior projects. 


\section{NOMENCLATURE}

$a$

$a^{\prime}$

ANOVA

ASQ

$b$

$b^{\prime}$

BPNPV

CapEx

CAVE

$\mathrm{CDF}$

Constrained Budget

Estimation
Lower bound of truncated distributions

The lower cumulative probability of the truncated distribution

Attributional Style Questionaire

Exponential base in DCA; or upper bound of truncated distributions

The upper cumulative probability of the truncated distribution Best Possible NPV is the summation of the true NPV's of best projects that could be selected from a global portfolio, usually by selecting them based on the true IE's.

Capital Expenditure, dollars spent or committed at the beginning of the project or portfolio.

Content Analysis of Verbatim Eplanations

Cumulative distribution function

Portfolio selection process that will take a limited CapEx into account to limit the number of projects in a portfolio. The term "constrained" will always be used with this kind of estimation, not to be confused with Limited Resources Assessment.

DB

Directional Bias 
DCA

Decision Error

$D_{i}$

Disappointment

E\&P

ED

$\mathrm{ED} \% \mathrm{E}$

$\mathrm{ED} \% \mathrm{~T}$

EDE

$\mathrm{EDE} \% \mathrm{E}$

EDE\% \%

ELOT
Decline Curve Analysis

The difference between the realized value of the selected portfolio and the best portfolio that can be selected.

Yearly decline rate

The difference between the estimated value of the selected portfolio and realized one. Usually a positive value to show reduction. Sometimes a negative number to imply pleasant surprise.

Exploration and Petroleum Engineering

Expected Disappointment, the average over many Monte-Carlo iterations.

Expected Disappointment as a percentage of the Estimated expected value $(\mathrm{EV})$.

Expected Disappointment as a percentage of the True expected value $(\mathrm{EV})$.

Expected Decision Error, the average over many Monte-Carlo iterations.

Expected Decision Error as a percentage of the Estimated expected value $(\mathrm{EV})$.

Expected Decision Error as a percentage of the True expected value $(\mathrm{EV})$.

Extended Life Orientation Test 


\begin{tabular}{|c|c|}
\hline Erf & Gaussian error function \\
\hline $\operatorname{Erf}^{-1}$ & Inverse of the Gaussian error function \\
\hline ERV & Expected Realized Value \\
\hline Estimated Project & The distribution of the possible outcomes of a project based on \\
\hline Distribution & Limited Resource Assessment. \\
\hline EstNPV & $\begin{array}{l}\text { Estimated NPV of a project or the total of the estimated NPV's of } \\
\text { several projects. }\end{array}$ \\
\hline EUR & Expected Ultimate Recovery \\
\hline $\mathrm{EV}$ & Expected Value \\
\hline$F_{X}$ & Cumulative distribution function \\
\hline$F_{X}^{-1}$ & Inverse of cumulative distribution function \\
\hline GIP & Gas In Place \\
\hline Global Portfolio & $\begin{array}{l}\text { Collection of oil and gas investment opportunities available to a } \\
\text { company or an individual. }\end{array}$ \\
\hline IE & Investment Efficiency $=\mathrm{NPV} / \mathrm{CapEx}$. \\
\hline IRR & Internal Rate of Return. \\
\hline $\begin{array}{l}\text { Limited Resources } \\
\text { Assessment }\end{array}$ & $\begin{array}{l}\text { Assessment that is constrained by time, money, or resources, } \\
\text { representing a typical industry study. }\end{array}$ \\
\hline $\ln$ & Natural logarithm \\
\hline $\operatorname{og}-\mathcal{N}$ & Lognormal distribution \\
\hline
\end{tabular}




\section{LOT}

LOT-R

NPV

O\&G

OC

OCF

OpEx

Portfolio

PVOCF

$\mathrm{q}_{\mathrm{i}}$

$q_{i}^{*}$

RF

RNPV

SD

TNPV

True Project
Life Orientation Test

Life Orientation Test-Revised

Net Present Value

Oil and Gas

Overconfidence

Operating Cash Flow is the revenue minus the operating expenditure without time-value calculations.

Expenditure relating to operation costs

Collection of oil and gas projects committed to be invested in.

Present Value of Operating Cash Flows. The discounted cash flow of all transactions related to the operating expenditure and revenue, except for CapEx.

Initial daily gas rate (Mscf/Day)

Pseudo initial daily gas rate, after correction for EUR (Mscf/Day)

Recovery factor

Realized NPV is the true NPV of a project or total true NPV's of projects that were selected.

Standard deviation

True NPV

The distribution of the possible outcomes of a project after doing 
Distribution

Unconstrained

Budget Estimation

Unlimited

Resources

Assessment

$\phi$

$\mu$

$\dot{\mu}$

$\sigma$

$\dot{\sigma}$ an unlimited-resource assessment of the project.

Portfolio selection process that is not constrained by CapEx when selecting number of projects in a portfolio.

Assessment that is not constrained by time, money, or resources.

However, the Unlimited Resources Assessment may not be used to run additional tests, acquire more data, or drill new wells.

Porosity

Mean of the specified distribution

Mean of the normal distribution whose logarithm will give desired lognormal distribution

Standard deviation of the specified distribution

Standard deviation of the normal distribution whose logarithm will give desired lognormal distribution 


\section{REFERENCES}

Armor, D.A. and Taylor, S.E. 2002. When Predictions Fail: The Dilemma of Unrealistic Optimism. In Heuristics and Biases: The Psychology of Intuitive Judgment., edition eds. Gilovich, T., Griffin, D., and Kahneman, D., New York, NY, US: Cambridge University Press. 334-347.

Atalaya, C.G. 2012. Optimism Syndrome in Business Life: A Research of Optimism About Academicians in Turkey. Procedia - Social and Behavioral Sciences 58 (0): 964-970.

Begg, S.H. and Bratvold, R.B. 2008. Systematic Prediction Errors in Oil and Gas Project and Portfolio Selection. Paper presented at the SPE Annual Technical Conference and Exhibition, Denver, Colorado, USA. SPE 116525-MS.

Begg, S.H., Bratvold, R.B., and Campbell, J.M. 2003. Shrinks or Quants: Who Will Improve Decision-Making. Paper presented at the SPE Annual Technical Conference and Exhibition, Denver, Colorado. Society of Petroleum Engineers 00084238 .

Ben-David, I., Graham, J.R., Harvey, C.R. et al. 2013. Managerial Miscalibration. Quarterly Journal of Economics 128 (4): 1547-1584.

Bickel, J.E. and Bratvold, R.B. 2007. Decision Making in the Oil \& Gas Industry: From Blissful Ignorance to Uncertainty-Induced Confusion In SPE Annual Technical Conference and Exhibition. Anaheim, California.

Bickel, J.E. and Bratvold, R.B. 2007. Decision Making in the Oil and Gas Industry: From Blissful Ignorance to Uncertainty-Induced Confusion. Paper presented at the SPE Annual Technical Conference and Exhibition, Anaheim, California, U.S.A. SPE 109610.

Block, R.A. and Harper, D.R. 1991. Overconfidence in Estimation: Testing the Anchoring-and-Adjustment Hypothesis. Organizational Behavior and Human Decision Processes 49 (2): 188-207.

Brashear, J.P., Becker, A.B., and Faulder, D.D. 2000. Where Have All the Profits Gone? Or, Evaluating Risk and Return of E\&P Projects. Paper presented at the SPE Annual Technical Conference and Exhibition, Dallas, Texas. 63056.

Brashear, J.P., Becker, A.B., and Faulder, D.D. 2001. Where Have All the Profits Gone? SPE Journal of Petroleum Technology 53 (6). 73141. 
Bratvold, R.B. and Begg, S.H. 2010. Making Good Decisions. Richardson, TX: Society of Petroleum Engineers.

Campbell, J.M.S., Campbell, J.M.J., and Campbell, R.A. 2001. Analyzing and Managing Risky Investments: JM Campbell.

Capen, E.C. 1976. The Difficulty of Assessing Uncertainty (Includes Associated Papers 6422 and 6423 and 6424 and 6425 ). SPE Journal of Petroleum Technology 28 (8): 843-850. 5579-PA.

Chang, E.C., Maydeu-Olivares, A., and D'Zurilla, T.J. 1997. Optimism and Pessimism as Partially Independent Constructs: Relationship to Positive and Negative Affectivity and Psychological Well-Being. Personality and Individual Differences 23 (3): 433-440.

Clemen, R.T. and Kwit, R.C. 2001. The Value of Decision Analysis at Eastman Kodak Company, 1990-1999. Interfaces 31 (5): 74-92.

Curry, L.A., Snyder, C., Cook, D.L. et al. 1997. Role of Hope in Academic and Sport Achievement. Journal of Personality and Social Psychology 73 (6): 1257.

Flyvbjerg, B. 2006. From Nobel Prize to Project Management: Getting Risks Right. Project Management Journal 37 (3): 5-15. 22149371.

Hawkins, J.T., Coopersmith, E.M., and Cunningham, P.C. 2002. Improving Stochastic Evaluations Using Objective Data Analysis and Expert Interviewing Techniques. Paper presented at the SPE Annual Technical Conference and Exhibition, San Antonio, Texas. SPE 77421.

James, S.A., Strogatz, D.S., Wing, S.B. et al. 1987. Socioeconomic Status, John Henryism, and Hypertension in Blacks and Whites. American Journal of Epidemiology 126 (4): 664-673.

Leach, P.E. 2006. Why Can't You Just Give Me the Number? Gainesville, Florida: Probabilistic Publishing.

Matlin, M.W. and Stang, D.J. 1978. The Pollyanna Principle: Selectivity in Language, Memory, and Thought: Schenkman Publishing Company Cambridge, MA.

Merrow, E.W. 2011. Oil Industry Megaprojects: Our Recent Track Record. 21858-MS.

Moore, D.A. and Healy, P.J. 2008. The Trouble with Overconfidence. Psychological Review 115 (2): 502. 
Oettingen, G. 1996. Positive Fantasy and Motivation. In The Psychology of Action: Linking Cognition and Motivation to Behavior, edition eds. Gollwitzer, P.M. and Bargh, J.A.: Guilford Press. 236-259.

Peterson, C. 1991. The Meaning and Measurement of Explanatory Style. Psychological Inquiry 2 (1): 1-10.

Peterson, C. 2000. The Future of Optimism. American Psychologist 55 (1): 44.

Peterson, C. and Park, C. 1998. Learned Helplessness and Explanatory Style. In Advanced Personality, edition: Springer. 287-310.

Peterson, C. and Seligman, M. 1984. Content Analysis of Verbatim Explanations: The Cave Technique for Assessing Explanatory Style. Unpublished manuscript, Virginia Polytechnic Institute and State University.

Peterson, C., Semmel, A., von Baeyer, C. et al. 1982. The Attributional Style Questionnaire. Cognitive Therapy and Research 6 (3): 287-299.

Puri, M. and Robinson, D.T. 2007. Optimism and Economic Choice. Journal of Financial Economics 86 (1): 71-99.

Radcliffe, N.M. and Klein, W.M. 2002. Dispositional, Unrealistic, and Comparative Optimism: Differential Relations with the Knowledge and Processing of Risk Information and Beliefs About Personal Risk. Personality and Social Psychology Bulletin 28 (6): 836-846.

Rose, P.R. 2004. Delivering on Our E\&P Promises. Leading Edge 23 (2): 165.

Scheier, M.E. and Carver, C.S. 1987. Dispositional Optimism and Physical Well-Being: The Influence of Generalized Outcome Expectancies on Health. Journal of Personality 55 (2): 169-210.

Scheier, M.F. and Carver, C.S. 1985. Optimism, Coping, and Health: Assessment and Implications of Generalized Outcome Expectancies. Health Psychology 4 (3): 219.

Scheier, M.F., Carver, C.S., and Bridges, M.W. 1994. Distinguishing Optimism from Neuroticism (and Trait Anxiety, Self-Mastery, and Self-Esteem): A Reevaluation of the Life Orientation Test. Journal of Personality and Social Psychology 67 (6): 1063-1078.prod.academic_MSTAR_614316796; 1995-07978-001. 
Scheier, M.F., Weintraub, J.K., and Carver, C.S. 1986. Coping with Stress: Divergent Strategies of Optimists and Pessimists. Journal of personality and social psychology 51 (6): 1257.

Schneider, S.L. 2001. In Search of Realistic Optimism: Meaning, Knowledge, and Warm Fuzziness. American Psychologist 56 (3): 250.

Smith, C.P. 1992. Motivation and Personality: Handbook of Thematic Content Analysis: Cambridge University Press.

Smith, J.E. and Winkler, R.L. 2006. The Optimizer's Curse: Skepticism and Postdecision Surprise in Decision Analysis. Management Science 52 (3): 311-322.

Snyder, C.R., Sympson, S.C., Ybasco, F.C. et al. 1996. Development and Validation of the State Hope Scale. Journal of personality and social psychology 70 (2): 321.

Spencer, S.M. and Norem, J.K. 1996. Reflection and Distraction Defensive Pessimism, Strategic Optimism, and Performance. Personality and Social Psychology Bulletin 22 (4): 354-365.

Taleb, N. 2007. The Black Swan : The Impact of the Highly Improbable. New York: Random House.

Taylor, S.E. 1989. Positive Illusions: Creative Self-Deception and the Healthy Mind: Basic Books.

Tversky, A. and Kahneman, D. 1974. Judgment under Uncertainty: Heuristics and Biases. Science 185 (4157): 1124-1131.

Virine, L. 2008. Judgment Elicitation Process for Multi-Criteria Decision-Making in Oil and Gas Industry. Paper presented at the International Petroleum Technology Conference, Kuala Lumpur, Malaysia. International Petroleum Technology Conference IPTC-12502-MS.

Weinstein, N.D. 1980. Unrealistic Optimism About Future Life Events. Journal of Personality and Social Psychology 39 (5): 806-820.

Welsh, M.B., Begg, S.H., and Bratvold, R.B. 2007. Modeling the Economic Impact of Cognitive Biases on Oil and Gas Decisions. Paper presented at the SPE Annual Technical Conference and Exhibition, Anaheim, California.

Welsh, M.B., Bratvold, R.B., and Begg, S.H. 2005. Cognitive Biases in the Petroleum Industry: Impact and Remediation. Paper presented at the SPE Annual Technical Conference and Exhibition, Dallas, Texas. SPE 96423. 